Research Article

\title{
Approximate Damped Oscillatory Solutions for Generalized KdV-Burgers Equation and Their Error Estimates
}

\author{
Weiguo Zhang and Xiang Li \\ School of Science, University of Shanghai for Science and Technology, Shanghai 200093, China \\ Correspondence should be addressed to Weiguo Zhang, zwgzwm@yahoo.com.cn
}

Received 27 April 2011; Revised 12 June 2011; Accepted 12 July 2011

Academic Editor: Josip E. Pečarić

Copyright (C) 2011 W. Zhang and X. Li. This is an open access article distributed under the Creative Commons Attribution License, which permits unrestricted use, distribution, and reproduction in any medium, provided the original work is properly cited.

We focus on studying approximate solutions of damped oscillatory solutions of generalized KdVBurgers equation and their error estimates. The theory of planar dynamical systems is employed to make qualitative analysis to the dynamical systems which traveling wave solutions of this equation correspond to. We investigate the relations between the behaviors of bounded traveling wave solutions and dissipation coefficient, and give two critical values $\lambda_{1}$ and $\lambda_{2}$ which can characterize the scale of dissipation effect, for right and left-traveling wave solution, respectively. We obtain that for the right-traveling wave solution if dissipation coefficient $\alpha \geq \lambda_{1}$, it appears as a monotone kink profile solitary wave solution; that if $0<\alpha<\lambda_{1}$, it appears as a damped oscillatory solution. This is similar for the left-traveling wave solution. According to the evolution relations of orbits in the global phase portraits which the damped oscillatory solutions correspond to, we obtain their approximate damped oscillatory solutions by undetermined coefficients method. By the idea of homogenization principle, we give the error estimates for these approximate solutions by establishing the integral equations reflecting the relations between exact and approximate solutions. The errors are infinitesimal decreasing in the exponential form.

\section{Introduction}

Generalized KdV equation with dissipation term

$$
u_{t}+b u^{p} u_{x}-\alpha u_{x x}+u_{x x x}=0
$$

is the physical model describing the long-wave propagating in nonlinear media with dispersion-dissipation [1], where $\alpha \geq 0, b$ is any real number and $p$ is any positive integer. It is very important in theory and application. If $p=1,(1.1)$ becomes KdV-Burgers equation

$$
u_{t}+b u u_{x}-\alpha u_{x x}+u_{x x x}=0 .
$$


Equation (1.2) can be regarded as a control equation for many kinds of practical problems with some dissipation effect, such as shadow wave in viscous liquid, liquid flowing, and waving in elastic pipe, magnetosonic wave in plasma, and so on [2-8]. If $\alpha=0,(1.1)$ has no dissipation effect and becomes generalized $\mathrm{KdV}$ equation

$$
u_{t}+b u^{p} u_{x}+u_{x x x}=0
$$

Obviously, (1.1) is KdV equation [9-11] if $p=1$, while it is MKdV equation [12-15] if $p=2$. The stability of solitary wave solutions for (1.3) was studied in [16-22], and the results that the solitary wave solutions are stable if $p<4$ while that they are unstable if $p>4$ were obtained. Pego et al. summarized the discussion of $[4,20]$ in [1] and gave the following conclusion.

If $\alpha>0, c>0,(1.1)$ has a unique traveling wave solution $u(x, t)=\phi(x-c t)$ when $b=1$, where $\phi(\xi)$ satisfies

$$
\begin{gathered}
-c \phi+\frac{1}{p+1} \phi^{p+1}+\partial_{\xi}^{2} \phi=\alpha \partial_{\xi} \phi, \quad \xi \in R, \\
\phi(\xi) \longrightarrow \begin{cases}u_{L} & \xi \longrightarrow-\infty, \\
0 & \xi \longrightarrow+\infty,\end{cases}
\end{gathered}
$$

where $u_{L}=[c(p+1)]^{1 / p}$ is the unique positive solution of $-c u_{L}+(1 /(p+1)) u_{L}^{p+1}=0$. If $\alpha>2 \sqrt{p c}, \phi(\xi)$ monotonically decreases. If $\alpha<2 \sqrt{p c}, \phi(\xi)$ approaches $u_{L}$ in oscillatory form as $\xi \rightarrow-\infty$; while as $\xi \rightarrow+\infty, \phi(\xi)$ satisfies

$$
\phi(\xi) \sim e^{\nu\left(\xi-\xi_{0}\right)}, \quad \partial_{\xi} \phi(\xi) \sim v e^{\nu\left(\xi-\xi_{0}\right)},
$$

where $v=(1 / 2)\left(\alpha-\sqrt{\alpha^{2}+4 c}\right)<0$.

Studies in [1] focused on discussing the instability of oscillatory traveling wave solution of (1.1) when $b=1$ by the method of numerically simulating Evans' function $D(\lambda)$ and obtained that linear instability takes place when, (1) for fix positive $c$ and $p>4, \alpha$ is made sufficiently small; (2) for fix positive $\alpha$ and $p>4, c$ is made sufficiently large; (3) for fix positive $\alpha$ and $c, p$ is made sufficiently large.

About the damped oscillatory traveling wave solutions of (1.1) when $p=1$, there have been many references. From these references, we know that Grad and Hu [3] and Bona and Schonbek [4] obtained the existence of a damped oscillatory solution for KdVBurgers equation (1.2) by the method of planar dynamical systems, respectively. Besides the existence of a damped oscillatory solution for KdV-Burgers equation (1.2), Johnson also presented the asymptotic expansion for this solution in [5]. Canosa and Gazdag [23] gave the numerical solution for a damped oscillatory solution of KdV-Burgers equation (1.2). By using qualitative theory of ordinary differential equations, Gao and Guan [24] studied the bounded non trivial traveling wave solutions of KdV-Burgers equation (1.2). In the meantime, they proved that when the dissipation coefficient is greater than some value, that is, it satisfied (3.3) in [24], the traveling wave solution for (1.2) is monotone, and its properties are the same as the properties of the kink profile solitary wave solution of Burgers equation; when the dissipation coefficient is less than some value, that is, it satisfied (3.9) in 
[25], the traveling wave solution of (1.2) is a damped oscillatory solution which has a bell profile head. However, they did not present any analytic solution or approximate solution for (1.2). Xiong [25] obtained a kink profile solitary wave solution for KdV-Burgers equation. In [26], S. D. Liu and S. D. Liu obtained an approximate damped oscillatory solution to a saddle-focus kink profile solitary solution of KdV-Burgers equation, without giving its error estimate. However, it is very important to give its error estimate, or people will feel unreliable.

From the above references, we can see that there is not only theoretical but also practical significance to find damped oscillatory solutions. However, the above references did not give exact or approximate damped oscillatory solutions of (1.1) when $p \geq 1$, and we have not seen the references about it. In this paper, we focus on studying the relations between the behaviors of bounded traveling wave solutions and the dissipation coefficient, showing the reason why the damped oscillatory solutions take place and how to obtain the approximate damped oscillatory solutions and giving their error estimates. We will obtain all the results in [1, Theorem 1.1] when $p$ is natural number, as well as the existent number of bounded traveling wave solutions and relations between the behaviors of bounded traveling wave solutions and the dissipation coefficient $\alpha$ in the case of $b>0, c<0, b<0, c>0$, and $b<0, c<0$, respectively. More importantly, we will give approximate damped oscillatory solution and its error estimate in the case of $c>0,0<\alpha<2 \sqrt{p c}$ when $p$ is any natural number.

This paper is organized as follows. In Section 2, we carry out qualitative analysis for the planar dynamical system corresponding to (1.1). We present all global phase portraits of this planar dynamical system and give the existent conditions and number of bounded traveling wave solutions of (1.1). We obtain that, if $\alpha=0,(1.1)$ at most has two bell profile solitary wave solutions or two kink profile solitary wave solutions and that, if $\alpha \neq 0,(1.1)$ at most has two bounded traveling wave solutions (kink profile or oscillatory traveling wave solutions). In Section 3, we discuss the relations between the behaviors of bounded traveling wave solutions and the dissipation coefficient $\alpha$. We find out two critical values $\lambda_{1}=2 \sqrt{p c}$ and $\lambda_{2}=2 \sqrt{-c}$ and obtain that for the righttraveling wave of the equation, a bounded traveling wave solution appears as a monotones kink profile solitary wave solution if dissipation coefficient $\alpha \geq \lambda_{1}$, while it appears as a damped oscillatory wave if $0<\alpha<\lambda_{1}$; for the left-traveling wave of the equation, a bounded traveling wave solution appears as a monotones kink profile solitary wave solution if dissipation coefficient $\alpha \geq \lambda_{2}$, while it appears as a damped oscillatory wave if $0<\alpha<\lambda_{2}$. In Section 4 , the exact bell profile and kink profile solitary wave solutions of (1.1) without dissipation effect are presented. Furthermore, according to the evolution relation of solution orbits in global phase portraits, by undetermined coefficients method, we obtain approximate damped oscillatory solutions of (1.1). In Section 5, we study the error estimate between approximate damped oscillatory solutions and their exact solutions. The difficulty of this problem is that we only know the approximate damped oscillatory solutions, but do not know their exact solutions. To overcome it, we use some transformations and the idea of homogenization principle and then establish the integral equations reflecting the relations between the exact solutions and approximate damped oscillatory solutions. Thus, we give error estimates for the approximate solutions obtained in Section 4. We can see that the errors between the exact solutions and approximate damped oscillatory solutions we obtained by this method are infinitesimal decreasing in exponential form. 


\section{Qualitative Analysis to Bounded Traveling Wave Solutions of (1.1)}

Assume that $u(x, t)=u(\xi)=u(x-c t)$ is a traveling wave solution of $(1.1)$, and $u(\xi)$ satisfies

$$
-c u^{\prime}(\xi)+b u^{p}(\xi) u^{\prime}(\xi)-\alpha u^{\prime \prime}(\xi)+u^{\prime \prime \prime}(\xi)=0,
$$

where $c$ is the wave speed. Integrating the above equation once yields

$$
u^{\prime \prime}(\xi)-\alpha u^{\prime}(\xi)-c u(\xi)+\frac{b}{p+1} u^{p+1}(\xi)=g
$$

where $g$ is an integral constant. Owing that we focus on studying dissipation effect to the system, we assume that the traveling wave solutions we study satisfy

$$
u^{\prime}(\xi), u^{\prime \prime}(\xi) \longrightarrow 0, \quad|\xi| \longrightarrow \infty
$$

and the asymptotic values $C_{+}$and $C_{-}\left(C_{+}=\lim _{\xi \rightarrow+\infty} u(\xi), C_{-}=\lim _{\xi \rightarrow-\infty}(\xi)\right)$ satisfy

$$
\frac{b}{p+1} x^{p+1}-c x=0
$$

so under the hypothesis (2.3) and (2.4), the traveling wave solutions of (1.1) satisfy

$$
u^{\prime \prime}(\xi)-\alpha u^{\prime}(\xi)-c u(\xi)+\frac{b}{p+1} u^{p+1}(\xi)=0
$$

Remark 2.1. In the following discussion, we will always assume that the traveling wave solutions of (1.1) satisfy (2.3) and (2.4). system

Letting $x=u(\xi)$ and $y=u^{\prime}(\xi)$, then (2.5) can be reformulated as a planar dynamical

$$
\begin{gathered}
\frac{d x}{d \xi}=y \equiv P(x, y) \\
\frac{d y}{d \xi}=\alpha y+c x-\frac{b}{p+1} x^{p+1} \equiv Q(x, y) .
\end{gathered}
$$

On $(x, y)$ phase plane, the number of singular points of system (2.6) depends on the number of real roots of $f(x)=(b /(p+1)) x^{p+1}-c x=0$. Denote $x_{0}=0, x_{1}=[(c(p+1)) / b]^{1 / p}$, $x_{2}=-x_{1}$. It is easy to know the following results on the real roots of $f(x)=0$.

(1) If $b c>0$, when $p$ is an even integer, $f(x)$ has three different real roots $x_{0}, x_{1}$ and $x_{2}$; when $p$ is an odd integer, $f(x)$ has two different real roots $x_{0}$ and $x_{1}$.

(2) If $b c<0$, when $p$ is an even integer, $f(x)$ only has one real root $x_{0}$; when $p$ is an odd integer, $f(x)$ has two different real roots $x_{0}$ and $x_{1}$. 
Since system (2.6) has and only has one singular point when $b c<0$, and $p$ is an even integer, there does not exist traveling wave solutions in (1.1). We will not consider this case. We use

$$
J\left(x_{i}, 0\right)=\left(\begin{array}{cc}
0 & 1 \\
-f^{\prime}\left(x_{i}\right) & \alpha
\end{array}\right), \quad i=0,1,2
$$

to denote the Jacobian matrix of system (2.6) at singular points $P_{i}\left(x_{i}, 0\right)$. Therefore, determinant of $J\left(x_{i}, 0\right)$, that is, $\operatorname{det}\left(J\left(x_{i}, 0\right)\right)$, is $f^{\prime}\left(x_{i}\right), i=0,1,2$. Use $\Delta_{i}$ to denote the discriminant of characteristic equation at $P_{i}\left(x_{i}, 0\right), \Delta_{i}=\alpha^{2}-4 f^{\prime}\left(x_{i}\right), i=0,1,2$. It is easy to know $\Delta_{0}=\alpha^{2}+4 c, \Delta_{1}=\alpha^{2}-4 p c$; if $p$ is an even integer, $\Delta_{1}=\Delta_{2}$.

In the following, we employ the theory and method of planar dynamical systems [2729] to discuss the type of singular points of system (2.6) and give the global phase portraits.

\subsection{In the Case of $\alpha=0$}

In this case, system (2.6) has the first integral

$$
\begin{aligned}
& H(x, y) \\
& \quad \equiv y^{2}+\frac{x^{2}\left(2 b x^{p}-c(p+1)(p+2)\right)}{(p+1)(p+2)}=h, \quad h \in R .
\end{aligned}
$$

It is easy to see that $P_{0}$ is a saddle point if $c>0$ and that $P_{0}$ is a center if $c<0$. The types of singular points $P_{i}$ are shown as follows:

(1) $b>0, c>0$. (i) If $p$ is an even integer, system (2.6) has three singular points $P_{0}(0,0)$ and $P_{i}\left(x_{i}, 0\right), i=1,2$. Since $(2.8)$ holds and $\operatorname{det}\left(J\left(x_{i}, 0\right)\right)=f^{\prime}\left(x_{i}\right)=p c>0, P_{i}\left(x_{i}, 0\right)$, $i=1,2$ are centers. (ii) If $p$ is an odd integer, system (2.6) has two singular points $P_{0}(0,0)$ and $P_{1}\left(x_{1}, 0\right)$. Since $(2.8)$ holds and $\operatorname{det}\left(J\left(x_{1}, 0\right)\right)=f^{\prime}\left(x_{1}\right)=p c>0, P_{1}$ is a center;

(2) $b<0, c<0$. (i) If $p$ is an even integer, system (2.6) has three singular points $P_{0}(0,0)$ and $P_{i}\left(x_{i}, 0\right), i=1,2$. Since $f^{\prime}\left(x_{i}\right)<0, P_{i}\left(x_{i}, 0\right), i=1,2$ are saddle points. (ii) If $p$ is an odd integer, system (2.6) has two singular points $P_{0}(0,0)$ and $P_{1}\left(x_{1}, 0\right)$. Since $f^{\prime}\left(x_{1}\right)<0, P_{1}$ is a saddle point;

(3) $b>0, c<0$. If $p$ is an odd integer, system (2.6) has two singular points $P_{0}(0,0)$ and $P_{1}\left(x_{1}, 0\right)$. Since $f^{\prime}\left(x_{1}\right)<0, P_{1}$ is a saddle point;

(4) $b<0, c>0$. If $p$ is an odd integer, system (2.6) has two singular points $P_{0}(0,0)$ and $P_{1}\left(x_{1}, 0\right)$. Since $(2.8)$ holds and $f^{\prime}\left(x_{1}\right)>0, P_{1}$ is a center.

\subsection{In the Case of $\alpha>0$}

It is easy to see that $P_{0}$ is a saddle point if $c>0$, while that $P_{0}$ is an unstable singular point if $c<0$, where $P_{0}$ is an unstable node point if $\Delta_{0}>0$ and $P_{0}$ is an unstable focus point if $\Delta_{0}<0$. The types of singular points $P_{i}$ are shown as follows: 


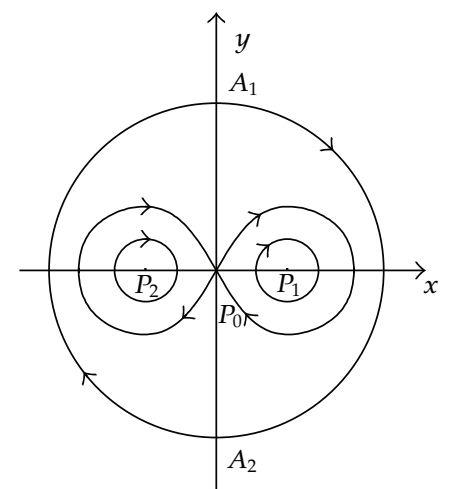

Figure 1: $(b>0, c<0)$.

(1) $b>0, c>0$. (i) If $p$ is an even integer, since $f^{\prime}\left(x_{i}\right)>0, P_{i}, i=1,2$ are unstable node points if $\Delta_{1}>0$, and $P_{i}, i=1,2$ are unstable focus points if $\Delta_{1}<0$. (ii) If $p$ is an odd integer, since $f^{\prime}\left(x_{i}\right)>0, P_{1}$ is unstable node point if $\Delta_{1}>0$ and is unstable focus point if $\Delta_{1}<0$;

(2) $b<0, c<0$. (i) If $p$ is an even integer, since $f^{\prime}\left(x_{i}\right)<0, P_{i}\left(x_{i}, 0\right), i=1,2$ are saddle points. (ii) If $p$ is an odd integer, since $f^{\prime}\left(x_{1}\right)<0, P_{1}$ is a saddle point;

(3) $b>0, c<0$. If $p$ is an odd integer, system (2.6) has two singular points $P_{0}(0,0)$ and $P_{1}\left(x_{1}, 0\right)$. Since $f^{\prime}\left(x_{1}\right)<0, P_{1}$ is a saddle point;

(4) $b<0, c>0$. If $p$ is an odd integer, system (2.6) has two singular points $P_{0}(0,0)$ and $P_{1}\left(x_{1}, 0\right)$. Since $f^{\prime}\left(x_{1}\right)>0, P_{1}$ is unstable node point if $\Delta_{1}>0$ and is unstable focus point if $\Delta_{1}<0$.

Applying Poincaré transformation to analyze singular points at infinity of system (2.6), it is clear that there only exists a couple of singular points $A_{i}, i=1,2$ at infinity on $y$ axis, where $A_{1}$ lies on the positive semiaxis of $y$ and $A_{2}$ lies on the negative semiaxis of $y$. There, respectively, exists a hyperbolic type region around $A_{i}$ if $p$ is an even integer and $b>0$. There, respectively, exists a elliptic type region around $A_{i}$ if $p$ is an even integer and $b<0$. There, respectively, exists a parabolic type region around $A_{i}$ if $p$ is an odd integer. Moreover, the circumference of Poincare disk is orbits.

For system (2.6), owing to $\partial P / \partial x+\partial Q / \partial y=\alpha$, by Bendixson-Dulac's criterion [27$29]$, the following proposition holds.

Proposition 2.2. If $\alpha>0$, then system (2.6) does not have any closed orbit or singular closed orbit with finite number of singular points on $(x, y)$ phase plane. Further, as $\alpha>0$, there exists no periodic traveling wave solution or bell profile solitary wave solution in (1.1).

According to the above analysis, we present the global phase portraits of system (2.6) under different parameter conditions (see Figures 1 and 12):

(1) global phase portraits in case of $\alpha=0$,

(i) $p$ is an even integer,

(ii) $p$ is an odd integer; 


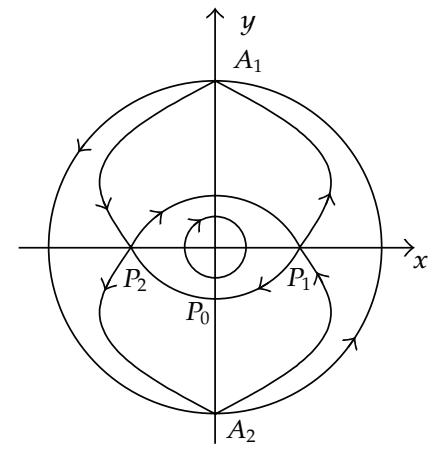

Figure 2: $(b<0, c<0)$.

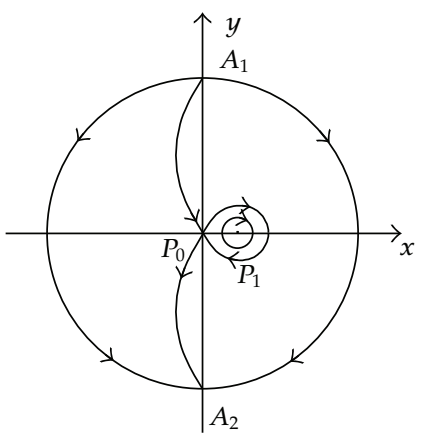

Figure 3: $(b>0, c>0)$.

(2) Global phase portraits in case of $\alpha>0$,

(i) $p$ is an even integer,

(ii) $p$ is an odd integer.

From Figures 1-12, we can derive the following propositions.

Proposition 2.3. (1) If $\alpha=0$, then except $P_{i}(i=0,1,2), L\left(P_{0}, P_{2}\right), L\left(P_{1}, P_{1}\right), L\left(P_{1}, P_{2}\right)$, and $L\left(P_{2}, P_{1}\right)$, the nonperiodic orbits of system (2.6) are unbounded. Moreover, the coordinate values of points on these orbits tend to infinity.

(2) If $\alpha>0$, then except $P_{i}(i=0,1,2)$ and $L\left(P_{i}, P_{0}\right), L\left(P_{0}, P_{i}\right)$, the nonperiodic orbits of system (2.6) are unbounded. Moreover, the coordinate values of points on these orbits tend to infinity.

Proof. (1) When $\alpha=0$, then except $P_{i} \quad(i=0,1,2), L\left(P_{0}, P_{2}\right), L\left(P_{1}, P_{1}\right), L\left(P_{1}, P_{2}\right)$, and $L\left(P_{2}, P_{1}\right)$, the nonperiodic orbits of system (2.6) are unbounded. Moreover, the coordinate values of points on these orbits tend to infinity. In fact, since these nonperiodic orbits either tend to $A_{1}$ or $A_{2}$ as $|\xi| \rightarrow+\infty$, the ordinate values on $y$ axis of these nonperiodic orbits must be unbounded.

Now, we prove that the abscissas of these orbits are unbounded by reduction to absurdity. Assume that the abscissas of these orbits are bounded. Since the tangent slope of the orbits at arbitrary point satisfies

$$
\frac{d y}{d x}=\frac{1}{y}\left(c x-\frac{b}{p+1} x^{p+1}\right)
$$




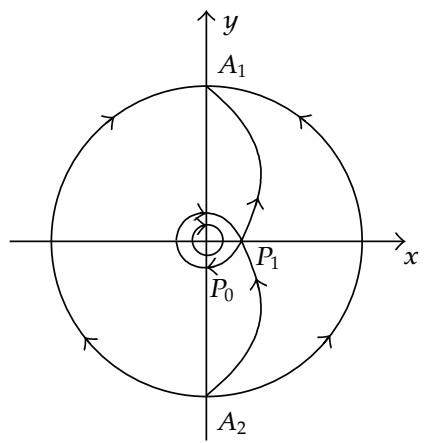

Figure 4: $(b<0, c<0)$.

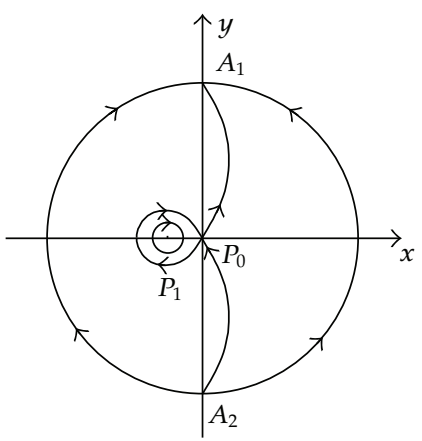

Figure 5: $(b<0, c>0)$.

$d y / d x \rightarrow 0$ as $|y| \rightarrow+\infty$. However, $d y / d x$ cannot keep bounded as $|y| \rightarrow+\infty$ according to the Differential Mean Value Theorem. Therefore, the abscissas of the orbits are unbounded.

(2) It can be proved similarly.

Proposition 2.4. (1) $\alpha=0$. If $p$ is an even integer, there exists two homoclinic orbits $L\left(P_{0}, P_{0}\right)$ or two heteroclinic orbits $L\left(P_{1}, P_{2}\right)$ and $L\left(P_{2}, P_{1}\right)$ in system (2.6) (see Figures 1 and 2$)$; if $p$ is an odd integer, there exists a unique homoclinic orbit $L\left(P_{0}, P_{0}\right)$ or $L\left(P_{1}, P_{1}\right)$ in system (2.6) (see Figures 3 and 6 ).

(2) $\alpha>0$. If $p$ is an even integer, there exists two heteroclinic orbits $L\left(P_{i}, P_{0}\right) \quad i=1,2$ in system (2.6) (see Figures 7 and 8); if $p$ is an odd integer, there exists a unique heteroclinic orbit $L\left(P_{0}, P_{1}\right)$ in system (2.6) (see Figures 9 and 12).

Considering that a homoclinic orbit or close orbit of a planar dynamical system corresponds to a bell profile solitary wave solution or periodic traveling wave solution of its corresponding nonlinear evolution equation and a heteroclinic orbit corresponds to a kink profile solitary wave solution or an oscillatory traveling wave solution, therefore, from Propositions 2.3 and 2.4 and Figures 1-12, we derive the following theorem.

Theorem 2.5. (1) $\alpha=0$. If $p$ is an even integer, (1.1) has two bell profile solitary wave solutions (corresponding to the homoclinic orbits $L\left(P_{0}, P_{0}\right)$ in Figure 1 ), or two kink profile solitary wave solutions (corresponding to the heteroclinic orbits $L\left(P_{2}, P_{1}\right)$ and $L\left(P_{1}, P_{2}\right)$ in Figure 2$)$; if $p$ is an odd integer, (1.1) has a unique bell profile solitary wave solution (corresponding to the homoclinic orbit in Figures 3-6). 


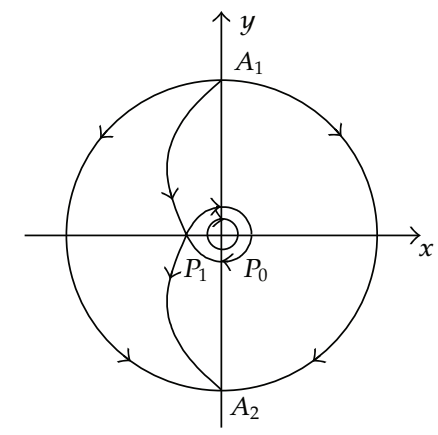

Figure 6: $(b>0, c<0)$.

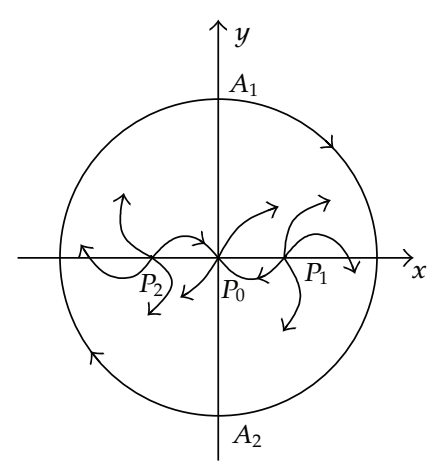

(a) $\left(\Delta_{1}>0\right)$

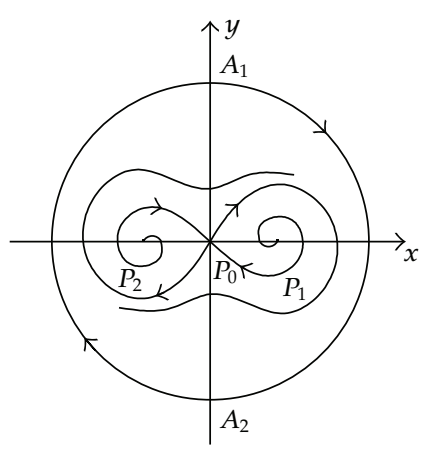

(b) $\left(\Delta_{1}<0\right)$

Figure 7: $(b>0, c>0)$.

(2) $\alpha>0$. If $p$ is an even integer, (1.1) has two bounded traveling wave solutions (corresponding to the heteroclinic orbits $L\left(P_{i}, P_{0}\right)$ or $L\left(P_{0}, P_{i}\right), \quad i=1,2$ in Figures 7 and 8); if $p$ is an odd integer, (1.1) has a unique bounded traveling wave solution (corresponding to the heteroclinic orbit in Figures 9-12).

\section{Relations between the Behaviors of Bounded Traveling Wave Solutions and Dissipation Coefficient $\alpha$ of (1.1)}

We firstly consider the case of wave speed $c>0$.

Theorem 3.1. Suppose that $p$ is an even integer, $b>0$, and wave speed $c>0$.

(1) If $\alpha>2 \sqrt{p c}$, (1.1) has a monotonically decreasing kink profile solitary wave solution $u(\xi)$, satisfying $u(-\infty)=x_{1}, u(+\infty)=0$. Meanwhile, (1.1) has a monotonically increasing kink profile solitary wave solution $u(\xi)$, satisfying $u(-\infty)=x_{2}, u(+\infty)=0(u(\xi)$, resp., corresponds to the orbit $L\left(P_{i}, P_{0}\right), \quad i=1,2$ in Figure $\left.7(a)\right)$.

(2) If $0<\alpha<2 \sqrt{p c}$, (1.1) has a damped oscillatory traveling wave solution $u(\xi)$ satisfying $u(-\infty)=x_{1}$ and $u(+\infty)=0$. This solution has maximum at $\widehat{\xi}_{1}$. Moreover, it has monotonically decreasing property if $\xi>\widehat{\xi}_{1}$, while it has damped property if $\xi<\widehat{\xi}_{1}$. That is, 


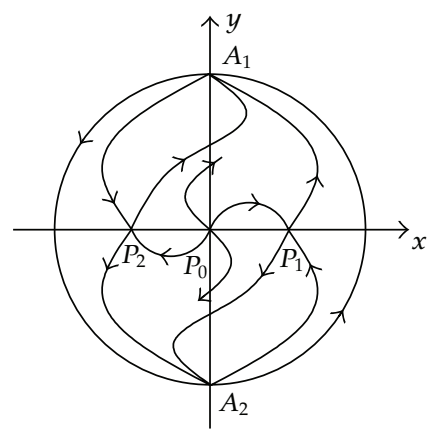

(a) $\left(\Delta_{0}>0\right)$

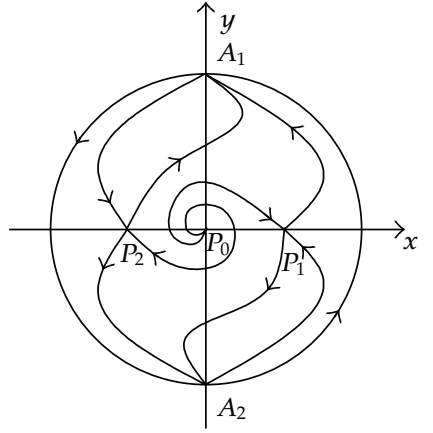

(b) $\left(\Delta_{0}<0\right)$

Figure 8: $(b<0, c<0)$.

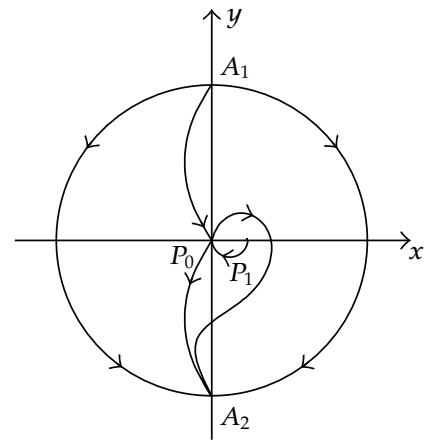

(a) $\left(\Delta_{1}>0\right)$

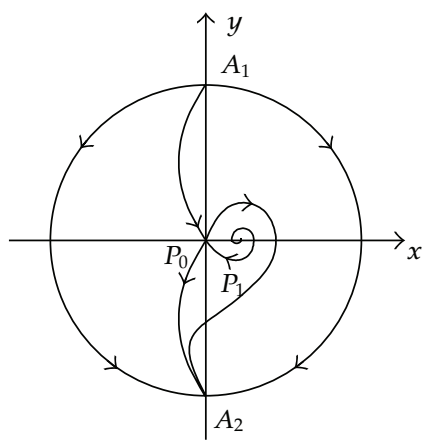

(b) $\left(\Delta_{1}<0\right)$

Figure 9: $(b>0, c>0)$.

there exist numerably infinite maximum points $\widehat{\xi}_{i}(i=1,2, \ldots,+\infty)$ and minimum points $\check{\xi}_{i}(i=1,2, \ldots,+\infty)$ on $\xi$ axis, such that

$$
\begin{gathered}
-\infty<\cdots<\check{\xi}_{n}<\widehat{\xi}_{n}<\cdots<\check{\xi}_{1}<\widehat{\xi}_{1}<+\infty, \\
\lim _{n \rightarrow \infty} \check{\xi}_{n}=\lim _{n \rightarrow \infty} \widehat{\xi}_{n}=-\infty, \\
u(+\infty)<u\left(\check{\xi}_{1}\right)<\cdots<u\left(\check{\xi}_{n}\right)<\cdots<u(-\infty)<\cdots<u\left(\widehat{\xi}_{n}\right)<\cdots<u\left(\widehat{\xi}_{1}\right), \\
\lim _{n \rightarrow \infty} u\left(\check{\xi}_{n}\right)=\lim _{n \rightarrow \infty} u\left(\check{\xi}_{n}\right)=-\infty, \\
\lim _{n \rightarrow \infty}\left(\check{\xi}_{n}-\check{\xi}_{n+1}\right)=\lim _{n \rightarrow \infty}\left(\check{\xi}_{n}-\check{\xi}_{n+1}\right)=\frac{4 \pi}{\sqrt{4 p c-\alpha^{2}}}
\end{gathered}
$$

hold. $u(\xi)$ corresponds to the orbit $L\left(P_{1}, P_{0}\right)$ in Figure $7(b)$.

Meanwhile, (1.1) has a damped oscillatory traveling wave solution $u(\xi)$ satisfying $u(-\infty)=$ $x_{2}$ and $u(+\infty)=0$. This solution has minimum at $\check{\xi}_{1}$. Moreover, it has damped property if $\xi<\check{\xi}_{1}$, while 


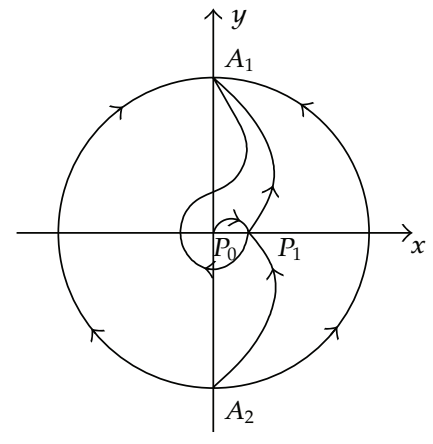

(a) $\left(\Delta_{0}>0\right)$

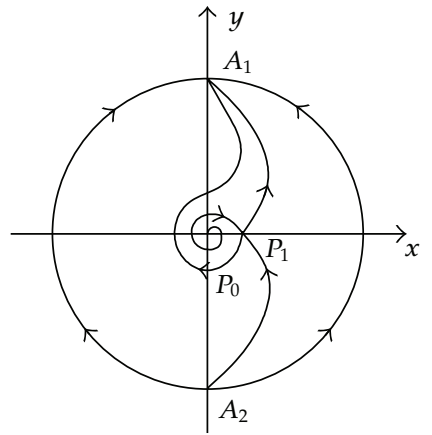

(b) $\left(\Delta_{0}<0\right)$

Figure 10: $(b<0, c<0)$.

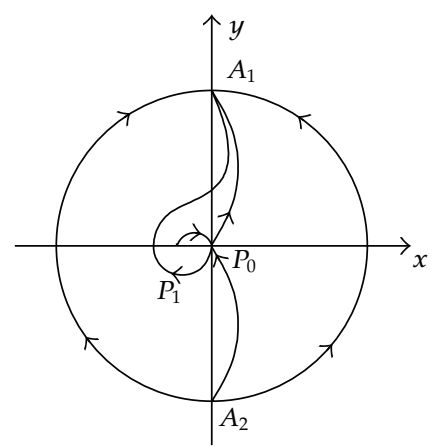

(a) $\left(\Delta_{1}>0\right)$

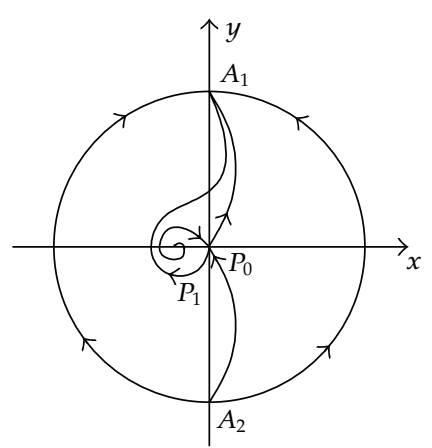

(b) $\left(\Delta_{1}<0\right)$

Figure 11: $(b<0, c>0)$.

it has monotonically increasing property if $\xi>\check{\xi}_{1}$. That is, there exists numerably infinite maximum points $\widehat{\xi}_{i}(i=1,2, \ldots,+\infty)$ and minimum points $\breve{\xi}_{i}(i=1,2, \ldots,+\infty)$ on $\xi$ axis, such that

$$
\begin{gathered}
-\infty<\check{\xi}_{1}<\widehat{\xi}_{1}<\cdots<\breve{\xi}_{n}<\widehat{\xi}_{n}<+\infty, \\
\lim _{n \rightarrow \infty} \check{\xi}_{n}=\lim _{n \rightarrow \infty} \widehat{\xi}_{n}=-\infty, \\
u(-\infty)<u\left(\widehat{\xi}_{1}\right)<\cdots<u\left(\widehat{\xi}_{n}\right)<u(+\infty)<u\left(\check{\xi}_{n}\right)<\cdots<u\left(\check{\xi}_{1}\right), \\
\lim _{n \rightarrow \infty} u\left(\widehat{\xi}_{n}\right)=\lim _{n \rightarrow \infty} u\left(\check{\xi}_{n}\right)=+\infty,
\end{gathered}
$$

and (3.3) hold. $u(\xi)$ corresponds to the orbit $L\left(P_{2}, P_{0}\right)$ in Figure $7(b)$.

Proof. We make use of the transformation $V(\xi)=\left(u(\xi)-x_{2}\right) /\left(x_{1}-x_{2}\right)$ to $(2.5)$ and note that $x_{2}=-x_{1}$ and $x_{1}=(c(p+1) / b)^{1 / p}$. Then we have

$$
V^{\prime \prime}(\xi)-\alpha V^{\prime}(\xi)+2^{p} c\left(V(\xi)-\frac{1}{2}\right)\left[\left(V(\xi)-\frac{1}{2}\right)^{p}-\left(\frac{1}{2}\right)^{p}\right]=0 .
$$




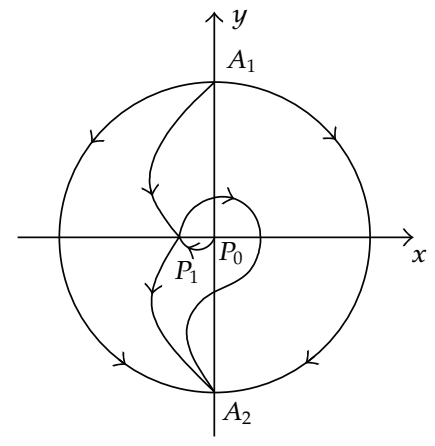

(a) $\left(\Delta_{0}>0\right)$

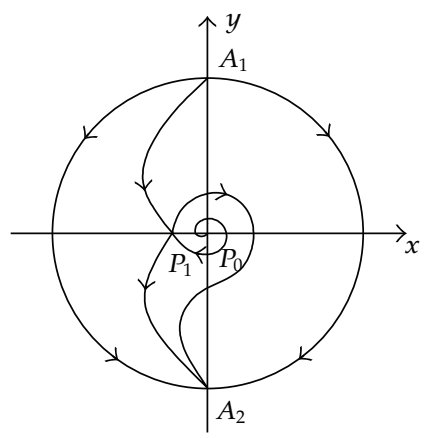

(b) $\left(\Delta_{0}<0\right)$

Figure 12: $(b>0, c<0)$.

If $p$ is an even integer, (3.5) is equivalent to

$$
\begin{aligned}
V^{\prime \prime}(\xi) & -\alpha V^{\prime}(\xi)+2^{p} c V(\xi)\left(V(\xi)-\frac{1}{2}\right)(V(\xi)-1) \\
\times & {\left[\left(V(\xi)-\frac{1}{2}\right)^{p-2}+\left(V(\xi)-\frac{1}{2}\right)^{p-4} \frac{1}{4}+\cdots+\left(\frac{1}{2}\right)^{p-2}\right]=0 . }
\end{aligned}
$$

Obviously, if $p$ is an even integer, $P_{1}^{\prime}(0,0), P_{2}^{\prime}(1 / 2,0)$, and $P_{3}^{\prime}(1,0)$ are the singular points of the system which (3.5) corresponds to. They correspond to singular points $P_{1}\left(x_{2}, 0\right), P_{2}(0,0)$, $P_{3}\left(x_{1}, 0\right)$ of system (2.6), respectively. Since the linear transformation keeps the properties of singular points, the results on $P_{i}, i=1,2,3$ given in Section 2 also hold for $P_{i}^{\prime}, i=1,2,3$ under corresponding conditions.

In the case that $p$ is an even integer and $c>0$, from the qualitative analysis carried out in Section 2, we have the fact that (2.5) does not have the bounded solution satisfying $u(-\infty)=u(+\infty)$. It only has the bounded solution satisfying the following alternative:
(A) $u(-\infty)=x_{1}, u(+\infty)=0$,
(B) $u(-\infty)=x_{2}, u(+\infty)=0$.

Namely, a bounded solution of (3.5) satisfies one of the following two cases:
$\left(\mathrm{A}_{1}\right) V(-\infty)=1, \quad V(+\infty)=1 / 2$,
$\left(\mathrm{B}_{1}\right) V(-\infty)=0, \quad V(+\infty)=1 / 2$.

We will use the following lemma in the proof.

Lemma 3.2. Assume that $f \in C^{1}[0,1], f(0)=f(1)=0, f^{\prime}(0)>0, f^{\prime}(1)<0$, and for all $u \in$ $(0,1), f(u)>0$ holds. Then there exists $r^{*}$ satisfying

$$
-2 \sqrt{\sup \frac{f(u)}{u}} \leq r^{*} \leq-2 \sqrt{f^{\prime}(0)}
$$


such that the necessary and sufficient condition under which problem

$$
\begin{gathered}
u^{\prime \prime}+r u^{\prime}+f(u)=0 \\
u(-\infty)=0, \quad u(+\infty)=1,
\end{gathered}
$$

has a monotone solution is $r \leq r^{*}$.

This lemma is quoted from [30-32]. Now we consider the solution of (3.5) which satisfies $\left(A_{1}\right)$.

Let $\omega=2(1-V)$, namely, $V=1-(1 / 2) \omega$. And then the solution of (3.5) which satisfies $\left(A_{1}\right)$ is equivalent to the solution of

$$
\begin{gathered}
\omega^{\prime \prime}-\alpha \omega^{\prime}+c(\omega-1)\left[(1-\omega)^{p}-1\right]=0 \\
\omega(-\infty)=0, \quad \omega(+\infty)=1,
\end{gathered}
$$

Assume $f(\omega)=c(\omega-1)\left[(1-\omega)^{p}-1\right]$, for all $\omega \in[0,1]$. It is easy to verify that $f(0)=0, f(1)=$ 0 . Since $f^{\prime}=c\left[(p+1)(1-\omega)^{p}-1\right], f^{\prime}(0)=p c, f^{\prime}(1)=-c<0$ and for all $\omega \in(0,1) \quad f(\omega)>0$ hold.

According to Lemma 3.2, there exists $r^{*}$ satisfying (3.7), such that, when $-\alpha \leq r^{*}$, (3.9) has a monotone solution. Since

$$
\left(\frac{f(\omega)}{\omega}\right)^{\prime}=\frac{\omega f^{\prime}(\omega)-f(\omega)}{\omega^{2}}=\frac{c\left[(1-\omega)^{p}(p \omega+1)-1\right]}{\omega^{2}}=\frac{c g(\omega)}{\omega^{2}}, \quad \forall \omega \in(0,1),
$$

where $g(\omega)=(1-\omega)^{p}(p \omega+1)-1$, for all $\omega \in[0,1]$. For all $\omega \in(0,1), g^{\prime}(\omega)=-p(p+1) \omega(1-$ $\omega)^{p-1}<0$ ( $p$ is an even integer). From $g(0)=0$, we have for all $\omega \in(0,1), g(\omega)<0$.

Therefore, for all $\omega \in(0,1),(f(\omega) / \omega)^{\prime}=c g(\omega) / \omega^{2}<0$, namely, $f(\omega) / \omega$ monotonically decreases in $(0,1)$. So

$$
\sup _{(0,1)} \frac{f(\omega)}{\omega}=\lim _{\omega \rightarrow 0} \frac{f(\omega)}{\omega}=\lim _{\omega \rightarrow 0} f^{\prime}(\omega)=p c
$$

According to (3.7) in Lemma 3.2, we know $r^{*}=-2 \sqrt{p c}$. So when $-\alpha \leq r^{*}=-2 \sqrt{p c}$, namely, $\alpha \geq 2 \sqrt{p c}$, there exists monotonically increasing solution in (3.9).

Since $V(\xi)=1-(1 / 2) \omega(\xi), u(\xi)=x_{2}+\left(x_{1}-x_{2}\right) V(\xi)$, if $\alpha \geq 2 \sqrt{p c}$, the traveling wave solution of $(1.1) u(\xi)$ satisfying condition $(A)$ monotonically decreases and appears as a monotone decreasing kink profile solitary wave solution.

Consider the solution of (3.5) satisfying condition $\left(B_{1}\right)$. Let $\omega=2 V$, namely, $V=$ $(1 / 2) \omega$. And then the solution of (3.5) which satisfies $\left(B_{1}\right)$ is equivalent to the solution of

$$
\begin{gathered}
\omega^{\prime \prime}-\alpha \omega^{\prime}+c(\omega-1)\left[(\omega-1)^{p}-1\right]=0 \\
\omega(-\infty)=0, \quad \omega(+\infty)=1,
\end{gathered}
$$




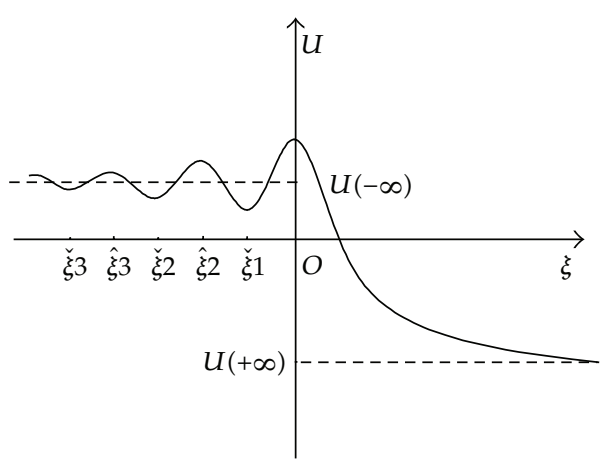

(a)

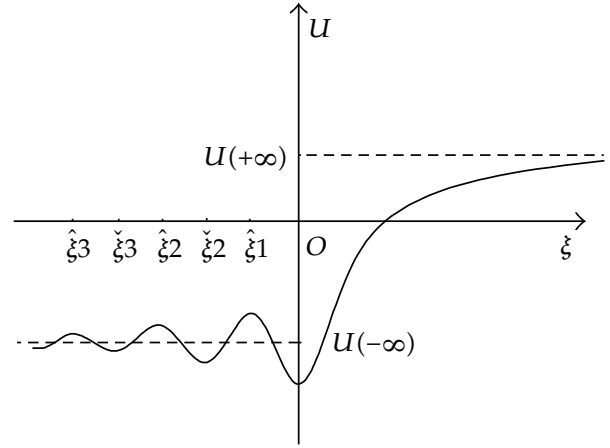

(b)

Figure 13: ( $p$ is an even integer, $b>0$, and wave speed $c>0,0<\alpha<2 \sqrt{p c}$ ).

Since (3.12) is the same as (3.9) when $p$ is an even integer, from the discussion about (3.9) we know there exists $r^{*}=-2 \sqrt{p c}$, such that when $-\alpha \leq r^{*}=-2 \sqrt{p c}$, namely, $\alpha \geq 2 \sqrt{p c}$, (3.12) has a monotonically increasing solution.

From $V(\xi)=(1 / 2) \omega(\xi)$, we can see that $V(\xi)$ increases when $\omega(\xi)$ increases. So if $\alpha \geq 2 \sqrt{p c}$, the traveling wave solution of $(1.1) u(\xi)$ satisfying condition $(B)$ monotonically decreases and appears as a monotonically decreasing kink profile solitary wave solution.

Next we will prove Theorem 3.1(2). Namely, if $0<\alpha<2 \sqrt{p c}$ the traveling wave solution of (1.1) is damped oscillatory. We take the traveling wave solution corresponding to focus-saddle orbit $L\left(P_{1}, P_{0}\right)$ in Figure $7(\mathrm{~b})$, for example, and for $L\left(P_{2}, P_{0}\right)$ in Figure $7(\mathrm{~b})$, it can be proved similarly. From the theory of planar dynamical systems we know that system (2.6) corresponding to (1.1) has three singular points on the plane, where $P_{0}(0,0)$ is a saddle point and $P_{1}\left(x_{1}, 0\right)$ and $P_{2}\left(x_{2}, 0\right)$ are unstable focus points. Moreover, $L\left(P_{1}, P_{0}\right)$ tends to $P_{1}$ spirally as $\xi \rightarrow-\infty$. The intersection points between $L\left(P_{1}, P_{0}\right)$ and $x$ axis at the right hand side of $P_{1}$ correspond to the maximum points of $u(\xi)$, while the ones at the left hand side of $P_{1}$ correspond to the minimum points of $u(\xi)$. Consequently, (3.1) and (3.2) hold. In addition, as $L\left(P_{1}, P_{0}\right)$ tends to $P_{1}$ sufficiently, its property is close to the property of linear approximate solution of system (2.6). Thus, the frequency of the orbit rotating around $P_{1}$ tends to $\sqrt{4 p c-\alpha^{2}} / 4 \pi$. And then, (3.3) holds.

Since a traveling wave solution $u(\xi)$ keeps the shape and speed unchanged when parallel shifting on $\xi$ axis, without loss of generality, we assume that $\widehat{\xi}_{1}=0$ and $\breve{\xi}_{1}=0$. The portraits of the oscillatory traveling waves described by Theorem 3.1(2) are shown in Figure 13.

We can prove Theorem 3.3 in the similar way.

Theorem 3.3. Suppose that $p$ is an odd integer and wave speed $c>0$.

(1) If $\alpha>2 \sqrt{p c}$, (1.1) has a monotone kink profile solitary wave solution $u(\xi)$, satisfying $u(-\infty)=x_{1}, u(+\infty)=0$. If $b>0, u(\xi)$ monotonically decreases, corresponding to the orbit $L\left(P_{1}, P_{0}\right)$ in Figure 9(a); if $b<0, u(\xi)$ monotonically increases, corresponding to the orbit $L\left(P_{1}, P_{0}\right)$ in Figure 11(a).

(2) If $0<\alpha<2 \sqrt{p c}$, (1.1) has a damped oscillatory traveling wave solution $u(\xi)$ satisfying $u(-\infty)=x_{1}$ and $u(+\infty)=0 . u(\xi)$ corresponds to the orbit $L\left(P_{1}, P_{0}\right)$ in Figure $9(b)$ and Figure 11(b), respectively. 
In the following, we consider the case of wave speed $c<0$. We can prove next two theorems.

Theorem 3.4. Suppose that $p$ is an even integer, $b<0$, and wave speed $c>0$.

(1) If $\alpha>2 \sqrt{-c}$, (1.1) has a monotonically increasing kink profile solitary wave solution $u(\xi)$, satisfying $u(-\infty)=0, u(+\infty)=x_{1}$. Meanwhile, (1.1) has a monotonically decreasing kink profile solitary wave solution $u(\xi)$, satisfying $u(-\infty)=0, u(+\infty)=x_{2}$. Here, $u(\xi)$, respectively, corresponds to the orbit $L\left(P_{0}, P_{i}\right), \quad i=1,2$ in Figure $8(a)$.

(2) If $0<\alpha<2 \sqrt{-c}$, (1.1) has a damped oscillatory traveling wave solution $u(\xi)$ satisfying $u(-\infty)=0$ and $u(+\infty)=x_{1}$. Meanwhile, (1.1) has an damped oscillatory traveling wave solution $u(\xi)$ satisfying $u(-\infty)=0$ and $u(+\infty)=x_{2}$. Here, $u(\xi)$ corresponds to the orbit $L\left(P_{0}, P_{i}\right), \quad i=1,2$ in Figure $8(b)$.

Theorem 3.5. Suppose that $p$ is an odd integer and wave speed $c<0$.

(1) If $\alpha>2 \sqrt{-c}$, (1.1) has a monotone kink profile solitary wave solution $u(\xi)$, satisfying $u(-\infty)=0, u(+\infty)=x_{1}$. If $b>0, u(\xi)$ monotonically decreases, corresponding to the orbit $L\left(P_{0}, P_{1}\right)$ in Figure 12(a); if $b<0, u(\xi)$ monotonically increases, corresponding to the orbit $L\left(P_{0}, P_{1}\right)$ in Figure 10(a).

(2) If $0<\alpha<2 \sqrt{-c}$, (1.1) has a damped oscillatory traveling wave solution $u(\xi)$ satisfying $u(-\infty)=0$ and $u(+\infty)=x_{1} . u(\xi)$ corresponds to the orbit $L\left(P_{0}, P_{1}\right)$ in Figures $10(b)$ and $12(b)$, respectively.

The way to prove Theorem 3.4 is the same to Theorem 3.5. For the case of $c<0$ and $\alpha>2 \sqrt{-c}$, the way to prove that traveling wave solution of (1.1) appears monotone is to utilize some transformations and Lemma 3.2; for the case of $c<0$ and $0<\alpha<2 \sqrt{-c}$, we can imitate the proof of Theorem 3.1(2) to prove the traveling wave solution of (1.1) appears damped oscillatory. Because in this case, $P_{0}$ is an unstable focus point, $P_{i}$ are saddle points and $L\left(P_{i}, P_{0}\right)$ is focus-saddle orbit. For clarity, we will prove that (1.1) has a monotonically decreasing kink profile solitary wave solution $u(\xi)$ satisfying $u(-\infty)=0, u(+\infty)=x_{1}$ in the case of $c<0, \alpha>2 \sqrt{-c}, \quad b>0$.

Firstly, according to Theorem 2.5, the unique bounded traveling wave solution of (1.1) $u(\xi)$ satisfies

$$
u(-\infty)=0, \quad u(+\infty)=x_{1} .
$$

Substitute $v(\xi)=\left(u(\xi)+x_{1}\right) /\left(2 x_{1}\right)$ to $(2.5)$. Then (2.5) and (3.13) become

$$
\begin{gathered}
v^{\prime \prime}-\alpha v^{\prime}+2^{p} c\left(v-\frac{1}{2}\right)\left[\left(v-\frac{1}{2}\right)^{p}-\frac{1}{2}\right]=0 \\
v(-\infty)=\frac{1}{2}, \quad v(+\infty)=1
\end{gathered}
$$


Let $\omega=2(v-1 / 2)$, namely, $v=(1 / 2)(\omega+1)$. Then (3.14) is equivalent to

$$
\begin{aligned}
& \omega^{\prime \prime}-\alpha \omega^{\prime}+c \omega\left(\omega^{p}-1\right)=0 \\
& \omega(-\infty)=0, \quad \omega(+\infty)=1 .
\end{aligned}
$$

Assume $f(\omega)=c \omega\left(\omega^{p}-1\right)$, for all $\omega \in[0,1]$. It is easy to verify that $f(\omega) \in$ $C^{1}[0,1], f(0)=0, f(1)=0$, and for all $\omega \in(0,1) f(\omega)>0$. Since $f^{\prime}(\omega)=c\left[(p+1) \omega^{p}-1\right]$, $f^{\prime}(0)=-c>0, f^{\prime}(1)=p c<0$. According to Lemma 3.2, there exists $r^{*}$ satisfying (3.7), such that when $-\alpha \leq r^{*}$, (3.15) has a monotone solution. Since for all $\omega \in(0,1)$

$$
\frac{f(\omega)}{\omega}=c\left(\omega^{p}-1\right), \quad\left(\frac{f(\omega)}{\omega}\right)^{\prime}=c p \omega^{p-1}<0,
$$

we know $f(\omega) / \omega$ monotonically decreases in $(0,1)$. So $\sup _{(0,1)}(f(\omega) / \omega)=\lim _{\omega \rightarrow 0}(f(\omega) / \omega)=$ $-c$. And from $f^{\prime}(0)=-c$ and (3.7), we can derive $r^{*}=-2 \sqrt{-c}$. According to Lemma 3.2, if $-\alpha \leq$ $-2 \sqrt{-c}$, that is, $\alpha \geq 2 \sqrt{-c}, \omega(\xi)$ monotonically decreases satisfying $\omega(-\infty)=0, \omega(+\infty)=1$. Furthermore, in the case of $\alpha \geq 2 \sqrt{-c}, v=(1 / 2)(\omega+1)$ also monotonically decreases. Since if $c<0, b>0, x_{1}=(c(p+1) / b)^{1 / p}<0$, and $u(\xi)=2 x_{1} v(\xi)-x_{1}$. We know if $p$ is an odd integer, $c<0$ and $b>0$, when $\alpha \geq 2 \sqrt{-c}, u(\xi)$ monotonically decreases and satisfies $u(-\infty)=$ $0, u(+\infty)=x_{1}$.

Synthesize Theorems 3.1-3.5, We obtain two critical values for generalized KdVBurges equation (1.1), where $\lambda_{1}=2 \sqrt{p c}$ and $\lambda_{2}=2 \sqrt{-c}$. For the right-traveling wave of (1.1), if dissipation effect is large, namely, $\alpha \geq \lambda_{1}$, the traveling wave solution of (1.1) appears as a monotone kink profile solitary wave; while if dissipation effect is small, namely, $0<\alpha<\lambda_{1}$, it appears as a damped oscillatory wave. For the left-traveling wave of (1.1), if dissipation effect is large, namely, $\alpha \geq \lambda_{2}$, it appears as a monotone kink profile solitary wave; while if dissipation effect is small, that is, $0<\alpha<\lambda_{2}$, it appears as a damped oscillatory wave. In the theorems of this paper, there are definite conclusions on monotonicity of traveling wave solution when we find critical values of the dissipation coefficient. However, in [1, Theorem 1.1] it did not include the case of $\alpha=2 \sqrt{p c}$.

\section{Solitary Wave Solutions and Approximate Damped Oscillatory Solutions of $(1.1)$}

\subsection{Bell Profile Solitary Solutions of (1.1)}

From [33, Theorem 1], we know that (1.1) has bell profile solitary wave solutions in the following forms.

Theorem 4.1. Suppose $\alpha=0$ and wave speed $c>0$.

(1) If $p$ is an even integer and $b>0,(1.1)$ has two bell solitary wave solutions $u_{1}^{ \pm}(\xi)=$ $\pm \sqrt[p]{\varphi_{1}(\xi)}$

$$
\varphi_{1}(\xi)=\frac{c(p+1)(p+2)}{2 b} \operatorname{sech}^{2}\left(\frac{p}{2} \sqrt{c}\left(\xi-\xi_{0}\right)\right) .
$$

(2) If $p$ is an odd integer, either $b>0$ or $b<0,(1.1)$ has bell solitary wave solutions $u_{1}(\xi)=$ $\sqrt[p]{\varphi_{1}(\xi)}$, where $\varphi_{1}(\xi)$ is given by (4.1). 
Remark 4.2. It is easy to prove that $u_{1}^{ \pm}(\xi)= \pm \sqrt[p]{\varphi_{1}(\xi)}$ given in Theorem 4.1(1) corresponds to two symmetrical homoclinic orbits $L\left(P_{0}, P_{0}\right)$ in Figure $1 ; u_{1}(\xi)=\sqrt[p]{\varphi_{1}(\xi)}$ given in Theorem 4.1(2) corresponds to the homoclinic orbit $L\left(P_{0}, P_{0}\right)$ in Figure 3 when $b>0$ and corresponds to the homoclinic orbit $L\left(P_{0}, P_{0}\right)$ in Figure 5 when $b<0$.

\subsection{Kink Profile Solitary Solutions of (1.1)}

From [33, Theorem 4], we know that (1.1) has kink profile solitary wave solutions in the following forms.

Theorem 4.3. Suppose wave speed $c<0$ :

$$
\varphi_{2}(\xi)=\frac{A}{\left(1+e^{-\delta\left(\xi-\xi_{0}\right)}\right)^{2}}=\frac{A}{2}\left(1-\tanh \frac{\delta}{2}\left(\xi-\xi_{0}\right)-\frac{1}{2} \operatorname{sech}^{2} \frac{\delta}{2}\left(\xi-\xi_{0}\right)\right),
$$

where $\alpha^{2}=-\left((p+4)^{2} c\right) /(2(p+2)), \delta=p \alpha /(p+4), A=-2 \alpha^{2}(p+1)(p+2) /\left(b(p+4)^{2}\right)$.

(1) If $p$ is an even integer and $b<0,(1.1)$ has two kink solitary wave solutions $u_{2}^{ \pm}(\xi)=$ $\pm \sqrt[p]{\varphi_{2}(\xi)} ;$

(2) If $p$ is an odd integer, either $b>0$ or $b<0$, (1.1) has kink solitary wave solutions $u_{2}^{+}(\xi)=$ $\sqrt[p]{\varphi_{2}(\xi)}$

Remark 4.4. Since $\alpha^{2}=-\left((p+4)^{2} c\right) /(2(p+2)) \geq 4(-c)$, that is, $\alpha \geq 2 \sqrt{-c}$, the conclusions in Theorem 4.3 are consistent to those in Theorems 3.4 and 3.5, and the conclusions are the concrete realization of Theorems 3.4 and 3.5 when $\alpha \geq \sqrt{-c} \cdot u_{2}^{ \pm}(\xi)= \pm \sqrt[p]{\varphi_{2}(\xi)}$ given in Theorem 4.3(1) corresponds to two heteroclinic orbits $L\left(P_{0}, P_{i}, \quad i=1,2\right)$ in Figure 8(a); $u_{2}^{+}(\xi)=\sqrt[p]{\varphi_{2}(\xi)}$ given in Theorem 4.3(2) corresponds to the heteroclinic orbit $L\left(P_{0}, P_{1}\right)$ in Figure 10 when $b<0$ and corresponds to the heteroclinic orbit $L\left(P_{0}, P_{1}\right)$ in Figure 12(a) when $b>0$.

By using undetermined coefficients method, we can derive Theorem 4.5 about MKdV equation in the case of $\alpha=0, p=2$.

Theorem 4.5. Suppose $\alpha=0, p=2$. If $b<0, c<0$. MKdV equation has two symmetrical kink solitary wave solutions

$$
u_{3}^{ \pm}(\xi)= \pm \sqrt{\frac{3 c}{b}} \tanh \left[\sqrt{-\frac{c}{2}}\left(\xi-\xi_{0}\right)\right]
$$

$u_{3}^{ \pm}(\xi)$ given in Theorem 4.5 corresponds to two heteroclinic orbits $L\left(P_{2}, P_{1}\right)$ and $L\left(P_{1}, P_{2}\right)$ in Figure 2.

\subsection{Approximate Damped Oscillatory Solutions of (1.1)}

In this section we want to obtain approximate damped oscillatory solutions of (1.1) corresponding to the focus-saddle orbits in Figures $7(b), 9(b)$, and 11(b). We take the approximate damped oscillatory solutions of (1.1) corresponding to the focus-saddle orbits in Figure 7(b) as example, and other cases can be obtained similarly. 
By the theory of planar dynamical systems, it is easy to see that focus-saddle orbit $L\left(P_{1}, P_{0}\right)$ in Figure 7(b) comes from the break of right homoclinic orbit $L\left(P_{0}, P_{0}\right)$ in Figure 1 under the effect of dissipation term $-\alpha u_{x x}(\xi)$ (the dissipation coefficient $\alpha$ satisfies $0<\alpha<$ $2 \sqrt{p c}$ orbit $L\left(P_{2}, P_{0}\right)$ comes from the break of left homoclinic orbit $L\left(P_{0}, P_{0}\right)$ in Figure 1 under the effect of dissipation term). Hence, the nonoscillatory part of the damped oscillatory solution corresponding to $L\left(P_{1}, P_{0}\right)$ can be denoted by the bell profile solitary wave solution of the form

$$
u^{*}(\xi)=\sqrt[p]{\varphi_{1}(\xi)}, \quad \xi \in\left[\xi_{0},+\infty\right)
$$

which is obtained in Theorem 4.1(1), where $\varphi_{1}(\xi)$ is given by (4.1). To express the oscillatory part of this damped oscillatory solution approximatively, we use the following solution of the form

$$
u(\xi)=e^{\beta\left(\xi-\xi_{0}\right)}\left(A_{1} \cos \left(B\left(\xi-\xi_{0}\right)\right)-A_{2} \sin \left(B\left(\xi-\xi_{0}\right)\right)\right)+C, \quad \xi \in\left(-\infty, \xi_{0}\right),
$$

where $A_{1}, A_{2}, B, C, \alpha$ are undetermined constants. The reason why we chose (4.5) is that (4.5) has both damped and oscillatory properties since $e^{\beta\left(\xi-\xi_{0}\right)}$ has damped property and $\left(A_{1} \cos \left(B\left(\xi-\xi_{0}\right)\right)-A_{2} \sin \left(B\left(\xi-\xi_{0}\right)\right)\right)$ has oscillatory property.

Substituting (4.5) into (2.5) and neglecting the terms including $O\left(e^{\beta\left(\xi-\xi_{0}\right)}\right)$, we have

$$
\begin{gathered}
B^{2}=\beta^{2}-\alpha \beta-c+b C^{p}, \\
\beta=\frac{\alpha}{2}, \\
\frac{b}{p+1} C^{p}-c=0 .
\end{gathered}
$$

In order to derive approximate damped oscillatory solution of (1.1), there still requires some conditions to connect (4.4) and (4.5). Since the properties of solutions are unchangeable as translating on $\xi$ axis, we take $\xi_{0}=0$ as a connective point and choose

$$
\frac{d^{i}}{d \xi^{i}} u(0)=\frac{d^{i}}{d \xi^{i}} u^{*}(0), \quad i=0,1
$$

namely,

$$
A_{1}+C=u^{*}(0), \quad \beta A_{1}-A_{2} B=0
$$

as connective conditions. $\xi_{0}=0$ is the extremal point of the bell profile solitary wave solutions, thus $(d / d \xi) u^{*}(0)=0$ holds.

Since (4.5) tends to $x_{1}$ as $\xi \rightarrow-\infty$, thus $C=x_{1}$. Further,

$$
\begin{gathered}
B^{2}=-\frac{1}{4} \alpha^{2}+p c \\
A_{1}=\sqrt[p]{\varphi_{1}(0)}-x_{1}, \quad A_{2}=\frac{\beta A_{1}}{B}=\frac{\alpha A_{1}}{2 B} .
\end{gathered}
$$


The value of $A_{1} \cos (B \xi)-A_{2} \sin (B \xi)$ is the same, either value of $B$ is positive or negative. Without loss of generality, let $B>0$ throughout this paper.

According to above analysis, we have the following theorem.

Theorem 4.6. Suppose $0<\alpha<2 \sqrt{p c}, b>0$, and wave speed $c>0$.

(1) When $p$ is an even integer, (1.1) has a damped oscillatory solution corresponding to focussaddle orbit $L\left(P_{1}, P_{0}\right)$, whose approximate solution is

$$
u(\xi) \approx \begin{cases}\sqrt[p]{\varphi_{1}(\xi)}, & \xi \in[0,+\infty) \\ e^{(\alpha / 2) \xi}\left(A_{1} \cos (B \xi)-A_{2} \sin (B \xi)\right)+x_{1}, & \xi \in(-\infty, 0)\end{cases}
$$

where $\varphi_{1}(\xi)$ is given by (4.1), $B=(1 / 2) \sqrt{-\alpha^{2}+4 p c}, A_{1}=\sqrt[p]{\varphi_{1}(0)}-x_{1}, A_{2}=\alpha A_{1} / 2 B$.

(2) Similarly, (1.1) has a damped oscillatory solution corresponding to focus-saddle orbit $L\left(P_{2}, P_{0}\right)$, whose approximate solution is

$$
u(\xi) \approx \begin{cases}-\sqrt[p]{\varphi_{1}(\xi)}, & \xi \in[0,+\infty), \\ e^{(\alpha / 2) \xi}\left(A_{1} \cos (B \xi)-A_{2} \sin (B \xi)\right)-x_{1}, & \xi \in(-\infty, 0),\end{cases}
$$

where $\varphi_{1}(\xi)$ is given by $(4.1), B=(1 / 2) \sqrt{-\alpha^{2}+4 p c}, A_{1}=-\sqrt[p]{\varphi_{1}(0)}+x_{1}, A_{2}=\alpha A_{1} / 2 B$.

If $p$ is an odd integer and $c>0$, from Theorem 3.3 we know (1.1) has a unique damped oscillatory solution if $0<\alpha<2 \sqrt{p c}$. When $b>0$, it corresponds to orbit $L\left(P_{1}, P_{0}\right)$ in Figure 9(b); when $b<0$, it corresponds to orbit $L\left(P_{1}, P_{0}\right)$ in Figure 11(b). $L\left(P_{1}, P_{0}\right)$ comes from the break of homoclinic orbit $L\left(P_{0}, P_{0}\right)$ under the dissipation effect (the dissipation coefficient $\alpha$ satisfies $0<\alpha<2 \sqrt{p c}$. Either in case of $b>0, c>0$ or $b<0, c>0$, the solitary wave solution corresponding to the homoclinic orbit $L\left(P_{0}, P_{0}\right)$ has the same expression $u_{1}(\xi)=\sqrt[p]{\varphi_{1}(\xi)}\left(\varphi_{1}(\xi)\right.$ is given by (4.1)). So we can obtain Theorem 4.7 by the method deriving Theorem 4.6.

Theorem 4.7. Suppose $p$ is an odd integer and wave speed $c>0$. When $0<\alpha<2 \sqrt{p c}$ (1.1) has a unique damped oscillatory solution, whose approximate solution can be expressed by (4.10) ( $p$ is an odd integer in (4.10)), where $\varphi_{1}(\xi)$ is given by (4.1).

Synthesizing Theorems 4.6 and 4.7, we can obtain the corollary as follows.

Corollary 4.8. Suppose $p$ is any natural number and $c>0$. Equation (1.1) has a unique damped oscillatory solution, satisfying $u(-\infty)=x_{1}, u(+\infty)=0$. Its approximate solution can be expressed by (4.10). Particularly, when $p$ is an even integer, (1.1) also has a damped oscillatory solution, satisfying $u(-\infty)=x_{2}, u(+\infty)=0$. Its approximate solution can be expressed by (4.11).

Thus, we have given the approximate solutions of right-traveling damped oscillatory solutions of (1.1). For left-traveling wave, if we know the kink profile solitary wave solution corresponding to the symmetrical heteroclinic orbit in Figure 2 when $p$ is an even integer, and the bell profile solitary wave solution corresponding to the homoclinic orbit in Figures 4 and 6 when $p$ is an odd integer, we can get approximate damped oscillatory solutions corresponding to the focus-saddle orbits in Figures 8(b), 10(b), and 12(b) in the case of $c>0$ by the same method. 
Since we have obtained two symmetrical kink profile solitary wave solutions (Theorem 4.5) in the case of $\alpha=0, p=2, c<0$, we can imitate the case of $c>0$ to get the following theorem.

Theorem 4.9. Suppose $p=2, b<0$, and wave speed $c<0$. When $0<\alpha<2 \sqrt{-c}$, (1.1) has two damped oscillatory solutions corresponding to focus-saddle orbit $L\left(P_{0}, P_{1}\right)$ and $L\left(P_{0}, P_{2}\right)$. The approximate solution corresponding to focus-saddle orbits $L\left(P_{0}, P_{1}\right)$ is

$$
u(\xi) \approx \begin{cases}u_{3}^{+}(\xi), & \xi \in[0,+\infty), \\ -A_{2} e^{(\alpha / 2) \xi} \sin (B \xi), & \xi \in(-\infty, 0),\end{cases}
$$

where $u_{3}^{+}(\xi)$ is given by (4.3), $B=(1 / 2) \sqrt{-\alpha^{2}+4 c}, A_{2} B=-\sqrt{-c / 2}$. The approximate solution corresponding to $L\left(P_{0}, P_{2}\right)$ is

$$
u(\xi) \approx \begin{cases}u_{3}^{-}(\xi), & \xi \in[0,+\infty), \\ A_{2} e^{(\alpha / 2) \xi} \sin (B \xi), & \xi \in(-\infty, 0),\end{cases}
$$

where $u_{3}^{-}(\xi)$ is given by $(4.3), B=(1 / 2) \sqrt{-\alpha^{2}+4 c}, A_{2} B=\sqrt{-c / 2}$.

\section{Error Estimates of Approximate Damped Oscillatory Solutions of $(1.1)$}

In this section, we investigate error estimates between approximate damped oscillatory solutions and its exact solutions given in Section 4.3. We still take the approximate solution (4.10) and its exact solution corresponding to the focus-saddle orbit $L\left(P_{1}, P_{0}\right)$ in Figure 7 (b) as example. Other error estimates can be discussed similarly.

Substitute

$$
V(\xi)=\frac{u(\xi)-x_{1}}{-2 x_{1}}
$$

and $\xi=-\eta(\eta>0)$ into (2.5). Consequently, the problem of finding an exact damped oscillatory solution for (2.5), which satisfies

$$
u(0)=\sqrt[p]{\varphi_{1}(0)}, \quad u^{\prime}(0)=0
$$

is converted into solving the following initial value problem:

$$
\begin{gathered}
\bar{V}_{\eta \eta}(\eta)+\alpha \bar{V}_{\eta}(\eta)+c\left(\bar{V}(\eta)-\frac{1}{2}\right) \bar{f}(\eta)=0, \\
\bar{V}(0)=\frac{\sqrt[p]{\varphi_{1}(0)}-x_{1}}{-2 x_{1}}, \quad \bar{V}_{\eta}(0)=0,
\end{gathered}
$$

where $\bar{V}(\eta)=V(-\eta)=V(\xi), \quad \bar{f}(\eta)=(-2)^{p}\left[(\bar{V}(\eta)-(1 / 2))^{p}-(1 / 2)^{p}\right]$. 
Simplifying above initial value problem, it becomes

$$
\begin{gathered}
\bar{V}_{\eta \eta}(\eta)+\alpha \bar{V}_{\eta}(\eta)+p c \bar{V}(\eta)+c \bar{V}^{2}(\eta) \bar{h}(\bar{V}(\eta))=0 \\
\bar{V}(0)=\frac{\sqrt[p]{\varphi_{1}(0)}-x_{1}}{-2 x_{1}}, \quad \bar{V}_{\eta}(0)=0
\end{gathered}
$$

where $\bar{h}(\bar{V}(\eta))$ is the polynomial of $\bar{V}(\eta)$ with $p-1$ order, satisfying

$$
c\left(\bar{V}(\eta)-\frac{1}{2}\right) \bar{f}(\xi)=p c \bar{V}(\eta)+c \bar{V}^{2}(\eta) \bar{h}(\bar{V}(\eta))
$$

We use the principle of homogenization to solve the following initial value problem:

$$
\begin{gathered}
\tilde{V}_{\eta \eta}(\eta)+\alpha \tilde{V}_{\eta}(\eta)+p c \tilde{V}(\eta)=-c \bar{V}^{2}(\eta) \bar{h}(\bar{V}(\eta)) \\
\tilde{V}(0)=\frac{\sqrt[p]{\varphi_{1}(0)}-x_{1}}{-2 x_{1}}, \quad \tilde{V}_{\eta}(0)=0
\end{gathered}
$$

where $\bar{V}(\eta)$ satisfies the initial value problem (5.4). It is easy to prove that the following two lemmas hold.

Lemma 5.1. Suppose that $\tilde{V}_{1}(\eta)$ and $\tilde{V}_{2}(\eta)$ are solutions of the initial value problems

$$
\begin{gathered}
\tilde{V}_{\eta \eta}(\eta)+\alpha \tilde{V}_{\eta}(\eta)+p c \tilde{V}(\eta)=0, \\
\tilde{V}(0)=\frac{\sqrt[p]{\varphi_{1}(0)}-x_{1}}{-2 x_{1}}, \quad \tilde{V}_{\eta}(0)=0, \\
\tilde{V}_{\eta \eta}(\eta)+\alpha \tilde{V}_{\eta}(\eta)+p c \tilde{V}(\eta)=-c \bar{V}^{2}(\eta) \bar{h}(\bar{V}(\eta)), \\
\tilde{V}(0)=0, \quad \tilde{V}_{\eta}(0)=0,
\end{gathered}
$$

respectively, then $\tilde{V}_{1}(\eta)+\tilde{V}_{2}(\eta)$ is a solution of the initial value problem (5.6).

Lemma 5.2. Suppose that $\tilde{V}_{3}(\eta, \tau)$ is a solution of the initial value problem

$$
\begin{gathered}
\tilde{V}_{\eta \eta}(\eta)+\alpha \tilde{V}_{\eta}(\eta)+p c \tilde{V}(\eta)=0, \\
\tilde{V}(0)=0, \quad \tilde{V}_{\eta}(\tau)=-c \bar{V}^{2}(\tau) \bar{h}(\bar{V}(\tau)), \quad \eta>\tau
\end{gathered}
$$

then $\int_{0}^{\eta} \tilde{V}_{3}(\eta, \tau) d \tau$ is a solution of initial value problem (5.8).

It is easy to obtain the solution of the initial value problem (5.7)

$$
\tilde{V}_{1}(\eta)=e^{\alpha_{1} \eta}\left(c_{1} \cos \left(\beta_{1} \eta\right)+c_{2} \sin \left(\beta_{1} \eta\right)\right)
$$

where $\alpha_{1}=-\alpha / 2, \beta_{1}=(1 / 2) \sqrt{4 p c-\alpha^{2}}, c_{1}=\left(\sqrt[p]{\varphi_{1}(0)}-x_{1}\right) /\left(-2 x_{1}\right), c_{2}=-\alpha_{1} c_{1} / \beta_{1}$. 
Let $t=\eta-\tau$, and substitute it in to the initial value problem (5.9). Then we have

$$
\tilde{V}_{3}(\eta, \tau)=\left(c / \beta_{1}\right) e^{\alpha_{1}(\eta-\tau)} \sin \left(\beta_{1}(\eta-\tau)\right) \bar{V}^{2}(\tau) \bar{h}(\bar{V}(\tau))
$$

So $\tilde{V}_{2}(\eta)=\left(c / \beta_{1}\right) \int_{0}^{\eta} e^{\alpha_{1}(\eta-\tau)} \sin \left(\beta_{1}(\eta-\tau)\right) \bar{V}^{2}(\tau) \bar{h}(\bar{V}(\tau)) d \tau$ is the solution of the initial value problem (5.8). Thus $\tilde{V}(\eta)=\tilde{V}_{1}(\eta)+\tilde{V}_{2}(\eta)$ is the solution of the initial value problem (5.6). Because the solution $\tilde{V}(\eta)$ of the initial value problem (5.6) satisfies the initial value problem (5.4), from the uniqueness of solutions, we have $\tilde{V}(\eta)=\bar{V}(\eta)$, namely,

$$
\bar{V}(\eta)=e^{\alpha_{1} \eta}\left(c_{1} \cos \left(\beta_{1} \eta\right)+c_{2} \sin \left(\beta_{1} \eta\right)\right)+\frac{c}{\beta_{1}} \int_{0}^{\eta} e^{\alpha_{1}(\eta-\tau)} \sin \left(\beta_{1}(\eta-\tau)\right) \bar{V}^{2}(\tau) \bar{h}(\bar{V}(\tau)) d \tau,
$$

where $\alpha_{1}=-\alpha / 2, \beta_{1}=(1 / 2) \sqrt{4 p c-\alpha^{2}}, c_{1}=\left(\sqrt[p]{\varphi_{1}(0)}-x_{1}\right) /-2 x_{1}, c_{2}=-\alpha_{1} c_{1} / \beta_{1}$.

Substituting $\eta=-\xi$ and (5.1) into (5.12) and making the transformation $t=-\tau$ in the above integral, then we have

$$
u(\xi)-x_{1}=e^{-\alpha_{1} \xi}\left(\bar{c}_{1} \cos \left(\beta_{1} \xi\right)+\bar{c}_{2} \sin \left(\beta_{1} \xi\right)\right)+\frac{c}{\beta_{1}} \int_{\xi}^{0} e^{-\alpha_{1}(\xi-t)} \sin \left(\beta_{1}(\xi-t)\right)\left(u(t)-x_{1}\right)^{2} h_{1}(t) d t
$$

where $h_{1}(t)=\left(1 / 2 x_{1}\right) \bar{h}(u(t))$ and $\bar{c}_{1}=-2 x_{1} c_{1}=\sqrt[p]{\varphi_{1}(0)}-x_{1}, \bar{c}_{2}=2 x_{1} c_{2}=\alpha_{1} \bar{c}_{1} / \beta_{1}$. Evidently, $\beta_{1}, \bar{c}_{1}, \bar{c}_{2}$ are equal to $B, A_{1},-A_{2}$ in Theorem 4.7(1), respectively. And $e^{-\alpha_{1} \xi}\left(\bar{c}_{1} \cos \left(\beta_{1} \xi\right)+\right.$ $\left.\bar{c}_{2} \sin \left(\beta_{1} \xi\right)\right)+x_{1}$ is the approximate damped oscillatory solution of (4.10). Therefore, (5.13) shows the relation between the exact damped oscillatory solution and the approximate damped oscillatory solution as $\xi<0$.

To derive the error estimate between approximate solution and exact solution of damped oscillatory solution corresponding to $L\left(P_{1}, P_{0}\right)$, we start from (5.12). Since damped oscillatory solution $u(\xi)$ is bounded and $\bar{V}(\eta)=V(\xi)=\left(u(\xi)-x_{1}\right) /-2 x_{1}$, there exists $M>0, M_{1}>0$ such that $|u(\xi)|<M,|\bar{V}(\eta)|<M_{1}$. Consequently, from (5.12), we have

$$
|\bar{V}(\eta)| \leq C_{1} e^{\alpha_{1} \eta}+\frac{c T_{2}}{\bar{\beta}_{1}} \int_{0}^{\eta} e^{\alpha_{1}(\eta-\tau)}|\bar{V}(\tau)| d \tau
$$

where $C_{1}=\left|c_{1}\right|+\left|c_{2}\right|, T_{2}=M_{1} T_{1}$, and $T_{1}$ is the supremum of $|\bar{h}(\bar{V}(\eta))|$. Since $\alpha_{1}<0$, for any $\eta_{1} \in(0, \eta]$, we have

$$
|\bar{V}(\eta)| \leq C_{1} e^{\alpha_{1} \eta_{1}}+\frac{c T_{2}}{\bar{\beta}_{1}} e^{\alpha_{1} \eta_{1}} \int_{0}^{\eta} e^{-\alpha_{1} \tau}|\bar{V}(\tau)| d \tau .
$$


By using Gronwall inequality, the above formula becomes

$$
|\bar{V}(\eta)| \leq C_{1} e^{\alpha_{1} \eta_{1}} \exp \left(-\frac{c T_{2}}{\alpha_{1} \beta_{1}} e^{\alpha_{1} \eta_{1}}\left(1-e^{-\alpha_{1} \eta}\right)\right) .
$$

Since $\eta_{1} \in(0, \eta]$ is chosen arbitrarily, letting $\eta_{1} \rightarrow \eta$, the above formula becomes

$$
|\bar{V}(\eta)| \leq C_{2} e^{\alpha_{1} \eta}
$$

where $C_{2}=C_{1} \exp \left(-c T_{2} / \alpha_{1} \beta_{1}\right)$

Substituting $\eta=-\xi$ and (5.1) into (5.17), we obtain

$$
\left|u(\xi)-x_{1}\right| \leq C_{3} e^{-\alpha_{1} \xi}, \quad \xi<0,
$$

where $C_{3}=2 C_{2} x_{1}$. (5.18) is the amplitude estimate of damped oscillatory solution of (1.1). From (5.18), it is obvious that $u(\xi)$ rapidly tends to $x_{1}$ as $\xi \rightarrow-\infty$.

From (5.12) and (5.17), we have

$$
\begin{aligned}
& \left|\bar{V}(\eta)-e^{\alpha_{1} \eta}\left(c_{1} \cos \left(\beta_{1} \eta\right)+c_{2} \sin \left(\beta_{1} \eta\right)\right)\right| \\
& \quad \leq \frac{c}{\beta_{1}} \int_{0}^{\eta} e^{\alpha_{1}(\eta-\tau)} \bar{V}^{2}(\tau)|\bar{h} \bar{V}(\tau)| d \tau \leq \frac{c T_{1} C_{2}^{2}}{\beta_{1}\left|\alpha_{1}\right|} e^{\alpha_{1} \eta}, \quad \eta>0 .
\end{aligned}
$$

Substituting $\eta=-\xi$ and (5.1) into (5.19), we have

$$
\left|u(\xi)-\left(e^{-\alpha_{1} \xi}\left(\bar{c}_{1} \cos \left(\beta_{1} \xi\right)+\bar{c}_{2} \sin \left(\beta_{1} \xi\right)\right)+x_{1}\right)\right| \leq T_{3} e^{-\alpha_{1} \xi}, \quad \xi<0,
$$

where $T_{3}=\left(2 x_{1} c T_{1} C_{2}^{2}\right) /\left(\beta_{1}\left|\alpha_{1}\right|\right)$. Equation (5.20) shows that the error estimate between the approximate solution (4.10) and its exact damped oscillatory solution is less than $\varepsilon_{1}(\xi)=$ $T_{3} e^{-\alpha_{1} \xi}$. Since $\varepsilon_{1}(\xi)=O\left(e^{(\alpha / 2) \xi}\right),(4.10)$ is meaningful to be an approximate solution of (1.1) when the conditions in Theorem 4.7(1) hold.

By using similar method, we can get error estimates between other approximate damped oscillatory solutions obtained above and their exact solutions. Their errors are all infinitesimals decreasing in the exponential form.

\section{Conclusion and Prospect}

In this paper, we make comprehensive qualitative analysis to the traveling wave solutions of generalized KdV-Burges equation (1.1) when $p$ is a natural number, study relations between the behaviors of bounded traveling wave solutions and dissipation coefficient $\alpha$, and obtain two critical values of dissipation coefficient: $\lambda_{1}=2 \sqrt{p c}$ and $\lambda_{2}=2 \sqrt{-c}$. For the righttraveling wave of the equation, if dissipation coefficient $\alpha \geq \lambda_{1}$, it appears as a monotonically kink profile solitary wave; if $0<\alpha<\lambda_{1}$, it appears as a damped oscillatory wave. For 
the left-traveling wave of the equation, if dissipation coefficient $\alpha \geq \lambda_{2}$, it appears as a monotonically kink profile solitary wave; if $0<\alpha<\lambda_{2}$, it appears as a damped oscillatory wave. According to the evolution relations of orbits in the global phase portraits which the damped oscillatory solutions correspond to, by using undetermined coefficients method, we obtain the approximate damped oscillatory solutions with a bell head and oscillatory tail, and the approximate damped oscillatory solutions with a kink head and oscillatory tail when $p=2$. Furthermore, by the idea of homogenization principle, we give the error estimates for these approximate solutions by establishing the integral equations reflecting the relations between approximate damped oscillatory solutions and their exact solutions. The errors are infinitesimal decreasing in the exponential form. It can be seen throughout this paper that we have obtained all the results in [1, Theorem 1.1] when $p$ is a natural number, as well as obtained the existent number of bounded traveling wave solutions and relations between the behaviors of bounded traveling wave solutions and the dissipation coefficient $\alpha$ in the case of $b>0, c<0, b<0, c>0$, and $b<0, c<0$, respectively. More importantly, we have got approximate damped oscillatory solution and its error estimate in the case of $c>0,0<\alpha<2 \sqrt{p c}$ when $p$ is any natural number.

According to Theorems 3.1, 3.3, and 4.7 and discussion in Section 5, we can give a corollary to the oscillatory solution of generalized KdV-Burgers equation (1.1) referring in $[1$, Theorem 1.1] if $b=1$.

Corollary 6.1. Suppose $p$ is a natural number, $c>0$, and dissipation coefficient satisfies $0<\alpha<$ $2 \sqrt{p c}$. Then generalized KdV-Burgers equation (1.1) has a unique oscillatory solution, which satisfies (1.4) if $b=1$, possessing the following properties:

(1) this solution corresponds to the orbit $L\left(P_{1}, P_{0}\right)$ in Figures $7(b), 9(b)$, and 11(b);

(2) this oscillatory solution is damped;

(3) this approximate oscillatory solution is

$$
u(\xi) \approx \tilde{u}(\xi)= \begin{cases}{\left[\frac{c(p+1)(p+2)}{2} \operatorname{sech}^{2}\left(\frac{p}{2} \sqrt{c} \xi\right)\right]^{1 / p},} & \xi \in[0,+\infty), \\ e^{(\alpha / 2) \xi}\left[A_{1} \cos (B \xi)-A_{2} \sin (B \xi)\right]+x_{1}, & \xi \in(-\infty, 0),\end{cases}
$$

where $A_{1}=((c(p+1)(p+2)) /(2))^{1 / p}-x_{1}, B=(1 / 2) \sqrt{-\alpha^{2}+4 p c}, A_{2}=A_{1} \alpha / 2 B ;$

(4) the error between the approximate oscillatory solution $\tilde{u}(\xi)$ and its exact damped oscillatory solution $u(\xi)$ is $\varepsilon(\xi)=O\left(e^{(\alpha / 2) \xi}\right),(\xi \rightarrow-\infty)$.

The following should be pointed out.

(1) This paper gives a method of finding approximate damped oscillatory solutions of nonlinear evolution equations with dissipation effect. Firstly, we make qualitative analysis to the equation. Secondly, we obtain its solitary wave solutions without dissipation effect. Finally, according to the evolution relations of orbits in the global phase portraits which the damped oscillatory solutions correspond to, we obtain its approximate damped oscillatory solutions. This method can also be applied to find approximate damped oscillatory solutions of other nonlinear evolution equations.

(2) Since we have not got the kink profile solitary wave solution corresponding to the heteroclinic orbits in Figure 2 when $p \neq 2$ and the bell profile solitary wave solution 
corresponding to the homoclinic orbit in Figures 4 and 6, we cannot obtain the damped oscillatory solutions with dissipation effect evolving from above orbits. This problem can be studied deeply in future.

\section{Acknowledgments}

This research is supported by the National Natural Science Foundation of China (no. 11071164), Shanghai Natural Science Foundation Project (no. 10ZR1420800), and Leading Academic Discipline Project of Shanghai Municipal Government (no. S30501).

\section{References}

[1] R. L. Pego, P. Smereka, and M. I. Weinstein, “Oscillatory instability of traveling waves for a KdVBurgers equation," Physica D, vol. 67, no. 1-3, pp. 45-65, 1993.

[2] D. J. Benney, “Long waves on liquid films," vol. 45, pp. 150-155, 1966.

[3] H. Grad and P. N. Hu, "Unified shock profile in a plasma," Physics of Fluids, vol. 10, no. 12, pp. 25962602, 1967.

[4] J. L. Bona and M. E. Schonbek, "Travelling-wave solutions to the Korteweg-de Vries-Burgers equation," Proceedings of the Royal Society of Edinburgh A, vol. 101, no. 3-4, pp. 207-226, 1985.

[5] R. S. Johnson, "A non-linear equation incorporating damping and dispersion," Journal of Fluid Mechanics, vol. 42, pp. 49-60, 1970.

[6] R. S. Johnson, "Shallow water waves on a viscous fluid-the undular bore," Physics of Fluids, vol. 15, no. 10, pp. 1693-1699, 1972.

[7] L. V. Wijngaarden, "On the motion of gas bubbles in a perfect fluid," Annual Review of Fluid Mechanics, vol. 4, pp. 369-373, 1972.

[8] P. N. Hu, "Collisional theory of shock and nonlinear waves in a plasma," Physics of Fluids, vol. 15, no. 5, pp. 854-864, 1972.

[9] D. J. Korteweg and G. de Vries, "On the change of form of long waves advancing in a rectangular channel, and on a new type of long stationary waves," Philosophical Magazine, vol. 39, pp. 422-443, 1895.

[10] C. S. Gardner, J. M. Greene, M. D. Kruskal, and R. M. Miura, "Method for solving the KortewegdeVries equation," Physical Review Letters, vol. 19, no. 19, pp. 1095-1097, 1967.

[11] C. S. Gardner, J. M. Greene, M. D. Kruskal, and R. M. Miura, "Korteweg-deVries equation and generalization. VI. Methods for exact solution," Communications on Pure and Applied Mathematics, vol. 27, pp. 97-133, 1974.

[12] C. S. Gardner, "Korteweg-de Vries equation and generalizations. IV. The Korteweg-de Vries equation as a Hamiltonian system," Journal of Mathematical Physics, vol. 12, pp. 1548-1551, 1971.

[13] K. Konno and Y. H. Ichikawa, "A modified Korteweg de Vries equation for ion acoustic waves," Journal of the Physical Society of Japan, vol. 37, no. 6, pp. 1631-1636, 1974.

[14] R. K. Dodd, J. C. Eilbeck, J. D. Gibbon, and H. C. Morris, Solitons and Nonlinear Wave Equations, Academic Press, London, UK, 1982.

[15] G. B. Whitham, Linear and Nonlinear Waves, Springer, New York, NY, USA, 1974, Pure and Applied Mathematic.

[16] E. K. Laedke and K. H. Spatscchek, "Stability theorem for Kdv Equations," Journal of Plasma Physics, vol. 101, no. 9, pp. 263-272, 1984.

[17] T. B. B. Iamin, "The stability of solitary wave," Proceedings of the Royal Society A, vol. 328, pp. 153-183, 1972.

[18] J. L. Bona, "On the stability theory of solitary waves," Proceedings of the Royal Society A, vol. 344, no. 1638, pp. 363-374, 1975.

[19] J. L. Bona, P. E. Souganidis, and W. A. Strauss, "Stability and instability of solitary waves of Kortewegde Vries type," Proceedings of the Royal Society A, vol. 411, no. 1841, pp. 395-412, 1987.

[20] R. L. Pego and M. I. Weinstein, "Eigenvalues, and instabilities of solitary waves," Philosophical Transactions of the Royal Society of London A, vol. 340, no. 1656, pp. 47-94, 1992.

[21] M. I. Wenistein, "Lyapunov stability of ground states of nonlinear dispersive evolution equations," Communications on Pure E Applied Mathematics, vol. 39, no. 1, pp. 51-68, 1986. 
[22] M. I. Weinstein, "Existence and dynamic stability of solitary wave solutions of equations arising in long wave propagation," Communications in Partial Differential Equations, vol. 12, no. 10, pp. 1133-1173, 1987.

[23] J. Canosa and J. Gazdag, "The Korteweg-de Vries-Burgers equation," Journal of Computational Physics, vol. 23, no. 4, pp. 393-403, 1977.

[24] G. Gao and K. Y. Guan, "Qualitative analysis to the traveling wave solutions of mixed type KdVBurgers equation," Science in China A, vol. 23, pp. 393-403, 1977 (Chinese).

[25] S. L. Xiong, "An analytic solution of Burgers-KdV equation," Chinese Science Bulletin, vol. 34, no. 14, pp. 1158-1162, 1989.

[26] S. D. Liu and S. K. Liu, "KdV-Burgers equation modelling of turbulence," Science in China A, vol. 35, no. 5, pp. 576-586, 1992.

[27] V. Nemytskii and V. Stepanov, Qualitative Theory of Differential Equations, Dover, New York, NY, USA, 1989.

[28] Z. F. Zhang, T. R. Ding, W. Z. Huang, and Z. X. Dong, Qualitative Theory of Differential Equations, vol. 101 of Translations of Mathematical Monographs, American Mathematical Society, Providence, RI, USA, 1992.

[29] Z. E. Ma and Y. C. Zhou, Qualitative and Stable Method of Ordinary Differential Equations, Science Press, Beijing, China, 1990.

[30] D. G. Aronson and H. F. Weinberger, "Multidimensional nonlinear diffusion arising in population genetics," Advances in Mathematics, vol. 30, no. 1, pp. 33-76, 1978.

[31] P. C. Fife, Mathematical Aspects of Reacting and Diffusing Systems, vol. 28 of Lecture Notes in Biomathematics, Springer, New York, NY, USA, 1979.

[32] Q. X. Ye and Z. Y. Li, Introduction of Reaction Diffusion Equations, Science Press, Beijing, China, 1990.

[33] W. G. Zhang, Q. S. Chang, and B. G. Jiang, "Explicit exact solitary-wave solutions for compound KdVtype and compound KdV-Burgers-type equations with nonlinear terms of any order," Chaos, Solitons and Fractals, vol. 13, no. 2, pp. 311-319, 2002. 


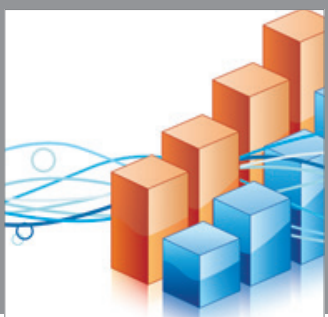

Advances in

Operations Research

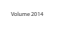

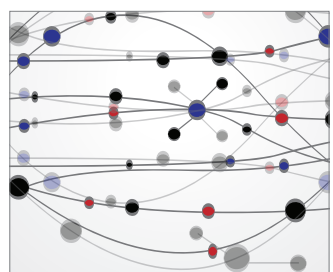

\section{The Scientific} World Journal
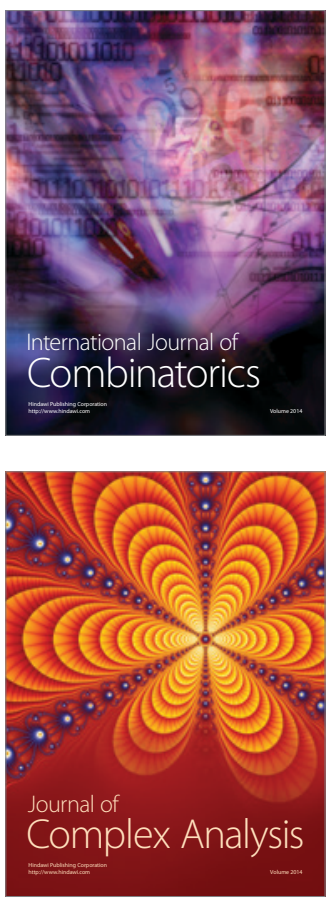

International Journal of

Mathematics and

Mathematical

Sciences
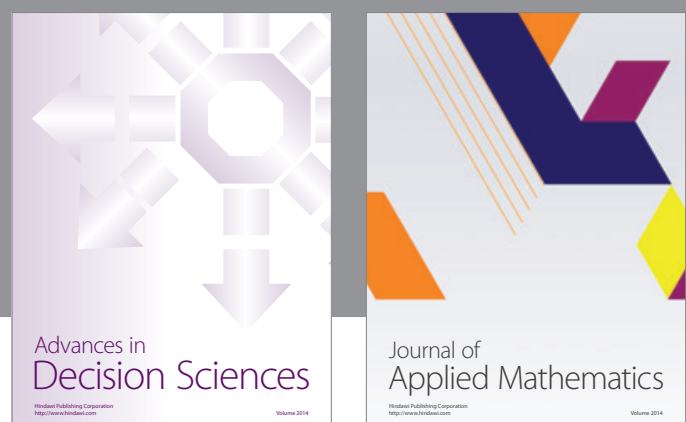

Journal of

Applied Mathematics
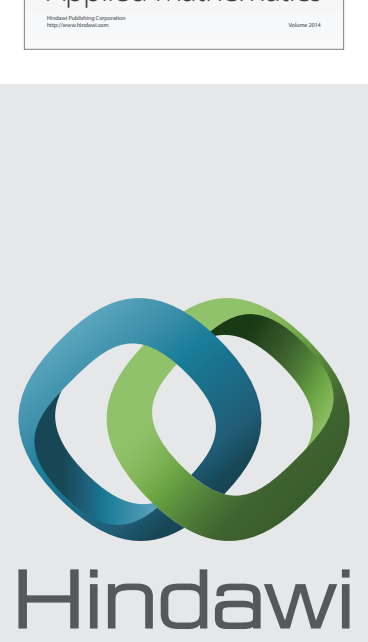

Submit your manuscripts at http://www.hindawi.com
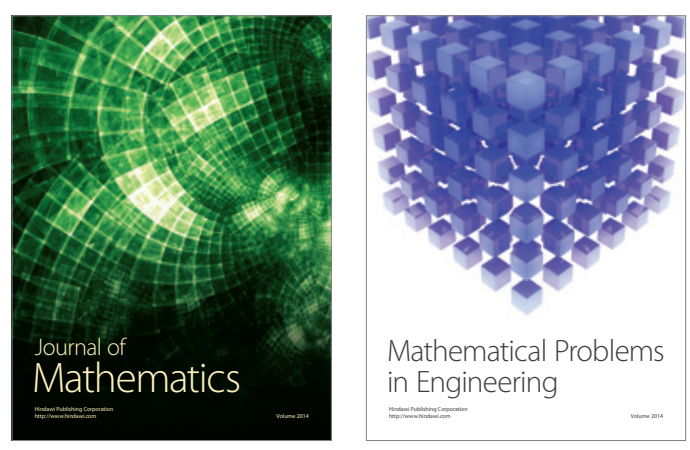

Mathematical Problems in Engineering
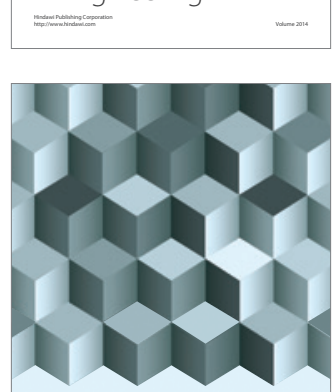

Journal of

Function Spaces
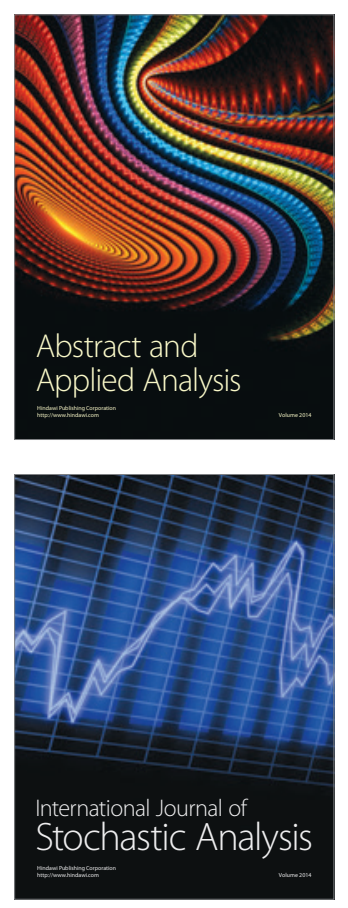

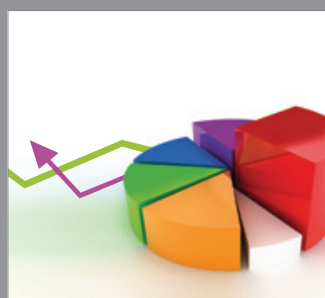

ournal of

Probability and Statistics

Promensencen
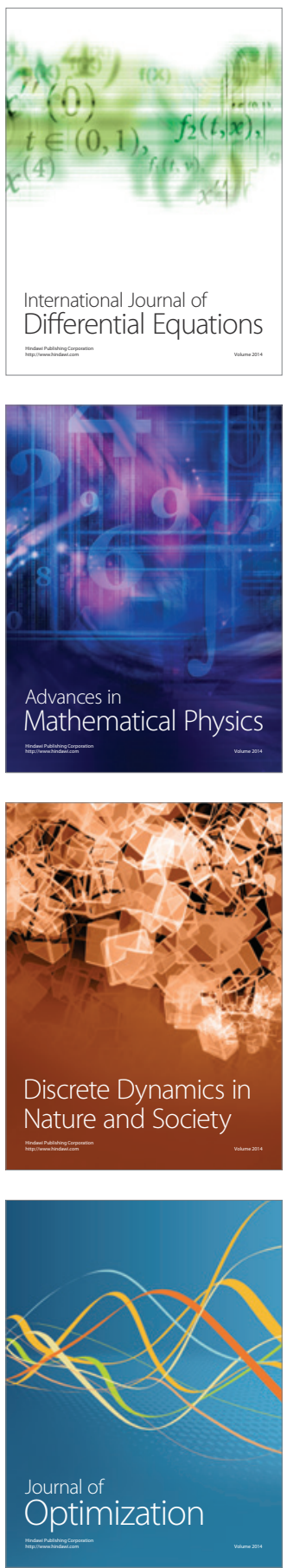